\title{
Nucleon and Delta masses in twisted mass chiral perturbation theory
}

\author{
André Walker-Loud* and Jackson M. S. WU \\ Department of Physics, University of Washington, Seattle, WA 98195-1560, USA
}

(Dated: July 16, 2018)

\begin{abstract}
We calculate the masses of the nucleons and deltas in twisted mass heavy baryon chiral perturbation theory. We work to quadratic order in a power counting scheme in which we treat the lattice spacing, $a$, and the quark masses, $m_{q}$, to be of the same order. We give expressions for the mass and the mass splitting of the nucleons and deltas both in and away from the isospin limit. We give an argument using the chiral Lagrangian treatment that, in the strong isospin limit, the nucleons remain degenerate and the delta multiplet breaks into two degenerate pairs to all orders in chiral perturbation theory. We show that the mass splitting between the degenerate pairs of the deltas first appears at quadratic order in the lattice spacing. We discuss the subtleties in the effective chiral theory that arise from the inclusion of isospin breaking.

PACS numbers: 12.38.Gc, 11.15.Ha, 12.39.Fe, 11.30.Rd
\end{abstract}

\section{INTRODUCTION}

Twisted mass lattice QCD (tmLQCD) 1, 2] is an alternative regularization for lattice QCD that has recently received considerable attention. ${ }^{1}$ It has the potential to match the attractive features of improved staggered fermions (efficient simulations [4], absence of "exceptional configurations" 1], $O(a)$ improvement at maximal twist [5], operator mixing as in the continuum [2, 6, 7]) while not sharing the disadvantage of needing to take roots of the determinant to remove unwanted degrees of freedom. Thus tmLQCD offers a promising and interesting new way to probe the properties and interactions of hadrons non-perturbatively from first principles.

Due to the limitations in computational capabilities, the quark masses, $m_{q}$, used in current simulations are still unphysically large. Thus extrapolations in $m_{q}$ are necessary if physical predications are to be made from lattice calculations. This can be done in a systematic and model independent way through the use of chiral perturbation theory $(\chi \mathrm{PT})$. Since $\chi \mathrm{PT}$ is derived in the continuum, it can be employed only after the continuum limit has been taken, where the lattice spacing, $a$, is taken to zero. However, when close to the continuum, it can be extended to lattice QCD at non-zero $a$, where discretization errors arising from the finite lattice spacing are systematically included in a joint expansion in $a$ and $m_{q}$. For tmLQCD with mass-degenerate quarks, the resulting "twisted mass chiral perturbation theory" $(\operatorname{tm} \chi \mathrm{PT})$ has been formulated previously [8, [9, 10], building on earlier work for the untwisted Wilson theory 11, 12, 13].

So far, $\operatorname{tm} \chi \mathrm{PT}$ has only been applied to the mesonic (pionic) sector. There have been studies on pion masses and decay constants for $m_{q} \gg a \Lambda_{\mathrm{QCD}}^{2}$ [8], the phase structure of tmLQCD for $m_{q} \sim a^{2} \Lambda_{\mathrm{QCD}}^{3}$ [9, 10, 14, 15, 16, 17], and quantities involving pions that do not involve final state interactions up to next-to-leading order (NLO) in the power counting scheme where $m_{q} \sim a \Lambda_{\mathrm{QCD}}^{2}$ [18]. However, as pointed out in Ref. [18], many of the pionic quantities considered are difficult to calculate in numerical simulations because they involve quark-disconnected diagrams. This motivated us to extend tm $\chi \mathrm{PT}$ to the baryon sector, which has heretofore not been done, enabling us to study analytically baryonic quantities that do not involve quark-disconnected diagrams. Numerical studies of the baryons in tmLQCD are already underway, and the first results from quenched simulations studying the nucleon and delta spectra have been obtained recently in Ref. [19].

In the baryon sector, the extension of $\chi \mathrm{PT}$ to the lattice at finite lattice spacing to $\mathcal{O}(a)$ [20], and to $\mathcal{O}\left(a^{2}\right)$ [21], has been done for a theory with untwisted Wilson fermions. We extend that work here to include the effects of "twisting", i.e. our starting underlying lattice theory is now tmLQCD. Specifically, we study the parity and flavor breaking effects due to twisting in the masses and mass splittings of nucleons and deltas in an $S U(2)$ chiral effective theory. The mass splittings are of particular interest to us as they allow one to quantify the size of the parity-flavor breaking effects in tmLQCD; furthermore, they present less difficulties to numerical simulations than their counterparts in the mesonic sector, which involve quark-disconnected diagrams.

\footnotetext{
*walkloud@u.washington.edu

†jmw@phys.washington.edu

${ }^{1}$ For a recent review see Ref. [3].
} 
We consider here tmLQCD with mass non-degenerate quarks [22], which includes an additional parameter, the mass splitting, $\epsilon_{q}$. This allows us to consider the theory both in and away from the strong isospin limit. With simulations in the near future most likely able to access the region where $m_{q} \sim a \Lambda_{\mathrm{QCD}}^{2}$, the power counting scheme we will adopt is

$$
1 \gg \varepsilon^{2} \sim a \Lambda_{\mathrm{QCD}} \sim \frac{m_{q}}{\Lambda_{\mathrm{QCD}}} \sim \frac{\epsilon_{q}}{\Lambda_{\mathrm{QCD}}}
$$

with $\varepsilon^{2}$ denoting the small dimensionless expansion parameters. In the following, we will work to $\mathcal{O}\left(\varepsilon^{4}\right)$ in this power counting.

The remainder of this article is organized as follows. In Sec. ЩA and IB we briefly review the definition of tmLQCD with mass non-degenerate quarks, and we show how the mass splitting can be included in the Symanzik Lagrangian and the $\mathcal{O}(a)$ meson chiral Lagrangian. Higher order corrections from the meson Lagrangian are not needed for the baryon observables to the order we work. In Sec. IC we extend the heavy baryon $\chi \mathrm{PT}(\mathrm{HB} \chi \mathrm{PT})$ to include the twisting effects to $\mathcal{O}\left(\varepsilon^{4}\right)$. In Sec. III we present the nucleon and delta masses in tm $\chi \mathrm{PT}$ in the strong isospin limit, including lattice discretization errors and the flavor and parity breaking induced by the twisted mass term. In Sec. IV we extend the calculation to include isospin breaking effects and discuss the subtleties that arise. We conclude in Sec. $\nabla$

\section{MASS NON-DEGENERATE TWISTED MASS CHIRAL PERTURBATION THEORY}

In this section, we work out the extension of the baryon chiral Lagrangian to $\mathcal{O}\left(a^{2}\right)$ given in Ref. 21] in tmLQCD. We start by briefly outlining the construction of the Symanzik Lagrangian in the mass non-degenerate case, which follows the same procedures as those in the mass-degenerate theory with minimal modifications.

\section{A. The effective continuum quark level Lagrangian}

The fermionic part of the Euclidean lattice action of tmLQCD with two mass non-degenerate quarks is

$$
S_{F}^{L}=\sum_{x} \bar{\psi}_{l}(x)\left[\frac{1}{2} \sum_{\mu} \gamma_{\mu}\left(\nabla_{\mu}^{\star}+\nabla_{\mu}\right)-\frac{r}{2} \sum_{\mu} \nabla_{\mu}^{\star} \nabla_{\mu}+m_{0}+i \gamma_{5} \tau_{1} \mu_{0}-\tau_{3} \epsilon_{0}\right] \psi_{l}(x),
$$

where we have written the action given in Ref. 22] for a general twist angle (not necessarily maximal), and in the so-called "twisted basis" [5]. The quark (flavor) doublets $\psi_{l}$ and $\bar{\psi}_{l}$ are the dimensionless bare lattice fields (with "l" standing for lattice and not indicating left-handed), and $\nabla_{\mu}$ and $\nabla_{\mu}^{*}$ are the usual covariant forward and backward dimensionless lattice derivatives, respectively. The matrices $\tau_{i}$ are the usual Pauli matrices acting in the flavor space, with $\tau_{3}$ the diagonal matrix. The bare normal mass, $m_{0}$, the bare twisted mass, $\mu_{0}$, and the bare mass splitting $\epsilon_{0}$, are all dimensionless parameters; an implicit identity matrix in flavor space multiplies the bare mass parameter $m_{0}$. The notation here is that both $m_{0}$ and $\epsilon_{0}$ are positive such that the upper component of the quark field is the lighter member of the flavor doublet with a positive bare mass.

Note that in the mass-degenerate case, twisting can be done using any of the $\tau_{i}$, the choice of $\tau_{3}$ is merely for convenience. Given the identity

$$
\exp \left(-i \frac{\pi}{4} \tau_{k}\right) \tau_{a} \exp \left(i \frac{\pi}{4} \tau_{k}\right)=\epsilon_{k a b} \tau_{b}
$$

one can always rotate from a basis where the twist is implemented by $\tau_{a}, a=1,2$, to a basis where it is implemented by $\tau_{3}$ using the vector transformation

$$
\bar{\psi} \rightarrow \bar{\psi} \exp \left(i \theta \tau_{k}\right), \quad \psi \rightarrow \exp \left(-i \theta \tau_{k}\right) \psi, \quad k=1,2,3, \quad \theta= \pm \frac{\pi}{4},
$$

where the appropriate sign for $\theta$ is determined by the index $a$. However, with $\tau_{3}$ used here to split the quark doublet so that the mass term is real and flavor-diagonal, it can not be used again for twisting if the fermionic determinant is to remain real. ${ }^{2}$

\footnotetext{
${ }^{2}$ One way to see this is to note that the mass terms $m_{0}+i \gamma_{5} \tau_{3} \mu_{0}-\tau_{3} \epsilon_{0}$ can be written as $\left(x_{0}-\tau_{3} y_{0}\right) \exp \left(i \alpha \gamma_{5}\right) \exp \left(i \beta \gamma_{5} \tau_{3}\right)$, where
} 
Following the program of Symanzik [23], and the same enumeration procedure detailed in Ref. [10], one can obtain the effective continuum Lagrangian at the quark level for mass non-degenerate quarks that describes the long distance physics of the underlying lattice theory. Its form is constrained by the symmetries of the lattice theory. To $\mathcal{O}\left(\varepsilon^{4}\right)$ in our power counting, in which we treat $a \Lambda_{\mathrm{QCD}}^{2} \sim m_{q} \sim \epsilon_{q}$, we find that the Pauli term is again the only dimension five symmetry breaking operator just as in the mass-degenerate case [10] (the details of this argument are provided in Appendix [-

$$
\mathcal{L}_{\text {eff }}=\mathcal{L}_{g}+\bar{\psi}\left(\not D+m+i \gamma_{5} \tau_{1} \mu-\epsilon_{q} \tau_{3}\right) \psi+b_{1} a \bar{\psi} i \sigma_{\mu \nu} F_{\mu \nu} \psi+O\left(a^{2}\right),
$$

where $\mathcal{L}_{g}$ is the continuum gluon Lagrangian, $m$ is the physical quark mass, defined in the usual way by

$$
m=Z_{m}\left(m_{0}-\widetilde{m}_{c}\right) / a,
$$

$\mu$ is the physical twisted mass

$$
\mu=Z_{\mu} \mu_{0} / a=Z_{P}^{-1} \mu_{0} / a
$$

and $\epsilon_{q}$ is the physical mass splitting

$$
\epsilon_{q}=Z_{\epsilon} \epsilon_{0} / a=Z_{S}^{-1} \epsilon_{0} / a .
$$

The factors $Z_{P}$ and $Z_{S}$ are matching factors for the non-singlet pseudoscalar and scalar densities respectively. Note that the lattice symmetries forbid additive renormalization to both $\mu_{0}$ and $\epsilon_{0}$ [22]. The quantity $\tilde{m}_{c}$ is the critical mass, aside from an $O(a)$ shift (see Ref. [18] and discussion below).

Anticipating the fact that the mesons contribute to the baryon masses only through loops, and so will be of $\mathcal{O}\left(\varepsilon^{3}\right)$ or higher, we only need to have a meson chiral Lagrangian to $\mathcal{O}(a)$ for the order we work; $\mathcal{L}_{\text {eff }}$ as given in Eq. (5) is sufficient for its construction. To build the effective chiral Lagrangian for baryons to $\mathcal{O}\left(\varepsilon^{4}\right)$ on the other hand, terms of $\mathcal{O}\left(a^{2}\right)$ in Eq. (5) are of the appropriate size to be included. However, except for the operator which breaks $\mathcal{O}(4)$ rotation symmetry, $a^{2} \bar{\psi} \gamma_{\mu} D_{\mu} D_{\mu} D_{\mu} \psi$, the $\mathcal{O}\left(a^{2}\right)$ operators do not break the continuum symmetries in a manner different than the terms explicitly shown in Eq. (5), and thus their explicit form is not needed. The $\mathcal{O}(4)$ breaking term will lead to operators in the baryon chiral Lagrangian at the order we work. However, it is invariant under twisting and thus contributes as those in the untwisted theory [21].

\section{B. The $S U(2)$ meson sector}

The low energy dynamics of the theory are described by a generalized chiral Lagrangian found by matching from the continuum effective Lagrangian (5). As usual, the chiral Lagrangian is built from the $S U(2)$ matrix-valued field $\Sigma$, which transforms under the chiral group $S U(2)_{L} \times S U(2)_{R}$ as

$$
\Sigma \rightarrow L \Sigma R^{\dagger}, \quad L \in S U(2)_{L}, R \in S U(2)_{R} .
$$

The vacuum expectation value, $\Sigma_{0}=\langle\Sigma\rangle$, breaks the chiral symmetry spontaneously down to an $S U(2)$ subgroup. The fluctuations around $\Sigma_{0}$ correspond to the pseudo-Goldstone bosons (pions).

From a standard spurion analysis, the chiral Lagrangian at $\mathcal{O}\left(\varepsilon^{2}\right)$ is (in Euclidean space ${ }^{3}$ )

$$
\mathcal{L}_{\chi}=\frac{f^{2}}{8} \operatorname{Tr}\left(\partial_{\mu} \Sigma \partial_{\mu} \Sigma^{\dagger}\right)-\frac{f^{2}}{8} \operatorname{Tr}\left(\chi^{\dagger} \Sigma+\Sigma^{\dagger} \chi\right)-\frac{f^{2}}{8} \operatorname{Tr}\left(\hat{A}^{\dagger} \Sigma+\Sigma^{\dagger} \hat{A}\right),
$$

where $f$ is the decay constant (normalized so that $f_{\pi}=132 \mathrm{MeV}$ ). The quantities $\chi$ and $\hat{A}$ are spurions for the quark masses and discretization errors respectively. At the end of the analysis they are set to the constant values

$$
\chi \longrightarrow 2 B_{0}\left(m+i \mu \tau_{1}-\epsilon_{q} \tau_{3}\right) \equiv \hat{m}+i \hat{\mu} \tau_{1}-\hat{\epsilon}_{q} \tau_{3}, \quad \hat{A} \longrightarrow 2 W_{0} a \equiv \hat{a},
$$

$x_{0} / y_{0}=\tan \beta / \tan \alpha$. Thus, the twisted mass term can be transformed away leaving just the normal mass term and the mass splitting term. However, since this involves an $U(1)$ axial transformation which is anomalous, an $i \alpha F \widetilde{F}$ term is introduced into the action which we see now is complex (because of the factor of $i$ ). Thus, since the gauge action is real, this means that the fermionic action (before the transformation) must be complex, and so the fermionic determinant obtained from it must also be complex. This also implies that a theory, where both the twist and the mass splitting are implemented by $\tau_{3}$, is $\alpha$-dependent.

${ }^{3}$ We will work in Euclidean space throughout this article. 
where $B_{0} \sim \mathcal{O}\left(\Lambda_{\mathrm{QCD}}\right)$ and $W_{0} \sim \mathcal{O}\left(\Lambda_{\mathrm{QCD}}^{3}\right)$ are unknown dimensionfull constants, and we have defined the quantities $\hat{m}, \hat{\mu}$ and $\hat{a}$.

As explained in Ref. [18], since the Pauli term transforms exactly as the quark mass term, they can be combined by using the shifted spurion

$$
\chi^{\prime} \equiv \chi+\hat{A}
$$

leaving the $\mathcal{O}\left(\varepsilon^{2}\right)$ chiral Lagrangian unchanged from its continuum form. This corresponds at the quark level to a redefinition of the untwisted component of the quark mass from $m$ to

$$
m^{\prime} \equiv m+a W_{0} / B_{0}
$$

This shift corresponds to an $O(a)$ correction to the critical mass, so that it becomes

$$
m_{c}=Z_{m} \tilde{m}_{c} / a-a W_{0} / B_{0}
$$

Since the $\mathcal{O}\left(\varepsilon^{2}\right)$ Lagrangian takes the continuum form, and the mass splitting term does not contribute at this order, the vacuum expectation value of $\Sigma$ at this order is that which cancels out the twist in the shifted mass matrix, exactly as in the mass-degenerate case:

$$
\langle 0|\Sigma| 0\rangle_{L O} \equiv \Sigma_{0}=\frac{\hat{m}+\hat{a}+i \hat{\mu} \tau_{1}}{M^{\prime}} \equiv \exp \left(i \omega_{0} \tau_{1}\right)
$$

where

$$
M^{\prime}=\sqrt{(\hat{m}+\hat{a})^{2}+\hat{\mu}^{2}} .
$$

Note that $M^{\prime}$ is the leading order result for the pion mass-squared, i.e. $m_{\pi}^{2}=M^{\prime}$ at $\mathcal{O}\left(\varepsilon^{2}\right)$. If we define the physical quark mass by

$$
m_{q}=\sqrt{m^{\prime 2}+\mu^{2}}
$$

then it follows from (15) that

$$
\cos \omega_{0}=m^{\prime} / m_{q}, \quad \sin \omega_{0}=\mu / m_{q} .
$$

Details of the non-perturbative determination of the twist angle and the critical mass can be found in 18], and will not be repeated here.

At $\mathcal{O}\left(\varepsilon^{4}\right)$, the mass non-degenerate chiral Lagrangian for the pions retains the same form as that in the mass degenerate case [10, 18], because the mass splitting does not induce any additional symmetry breaking operators in $\mathcal{L}_{\text {eff }}$. The $\mathcal{O}\left(\varepsilon^{4}\right)$ pion Lagrangian contains the usual Gasser-Leutwyler operators of $\mathcal{O}\left(\mathrm{m}^{2}, \mathrm{~m} p^{2}, p^{4}\right)$, where $\mathrm{m}$ is a generic mass parameter that can be $m, \mu$, or $\epsilon_{q}$, as well as terms of $\mathcal{O}\left(a \mathrm{~m}, a p^{2}, a^{2}\right)$ associated with the discretization errors. Now as we stated earlier, since the pions will enter only through loops in typical calculations of baryon observables, keeping the pion masses to $\mathcal{O}\left(\varepsilon^{4}\right)$ will lead to corrections of $\mathcal{O}\left(\varepsilon^{5}\right)$, which is beyond the order we work. As our concern is not in the meson sector, the $\mathcal{O}\left(\varepsilon^{2}\right)$ pion Lagrangian (10) is thus sufficient for our purpose in this work.

\section{The $S U(2)$ baryon sector}

With the effective continuum theory and the relevant part of the effective chiral theory describing the pions in hand, we now include the lowest lying spin- $\frac{1}{2}$ and spin- $\frac{3}{2}$ baryons into tm $\chi \mathrm{PT}$ by using $\mathrm{HB} \chi \mathrm{PT}[24$, 25, 26], which we will refer as the twisted mass $\mathrm{HB} \chi \mathrm{PT}(\operatorname{tmHB} \chi \mathrm{PT})$. In $S U(2)$ the spin- $\frac{1}{2}$ nucleons are described by a doublet

$$
N=\left(\begin{array}{l}
p \\
n
\end{array}\right)
$$

and the delta resonances form a flavor quartet. As they are spin- $\frac{3}{2}$, they are described by a Rarita-Schwinger field, $T_{\mu}^{i j k}$, which is totally symmetric in flavor and satisfies $\gamma_{\mu} T_{\mu}=0$. The delta fields are normalized such that

$$
T^{111}=\Delta^{++}, \quad T^{112}=\frac{1}{\sqrt{3}} \Delta^{+}, \quad T^{122}=\frac{1}{\sqrt{3}} \Delta^{0}, \quad T^{222}=\Delta^{-} .
$$


The free Lagrangian for the nucleons and deltas to $\mathcal{O}\left(\varepsilon^{2}\right)$ consistent with the symmetries of the lattice theory is (in Euclidean space)

$$
\begin{aligned}
\mathcal{L}_{\chi}= & \bar{N} i v \cdot D N-2 \alpha_{M} \bar{N} \mathcal{M}_{+}^{t w} N-2 \sigma_{M} \bar{N} N \operatorname{tr}\left(\mathcal{M}_{+}^{t w}\right)-2 \sigma_{W} \bar{N} N \operatorname{tr}\left(\mathcal{W}_{+}\right) \\
& +\left(\bar{T}_{\mu} i v \cdot D T_{\mu}\right)+\Delta\left(\bar{T}_{\mu} T_{\mu}\right)+2 \gamma_{M}\left(\bar{T}_{\mu} \mathcal{M}_{+}^{t w} T_{\mu}\right)-2 \bar{\sigma}_{M}\left(\bar{T}_{\mu} T_{\mu}\right) \operatorname{tr}\left(\mathcal{M}_{+}^{t w}\right)-2 \bar{\sigma}_{W}\left(\bar{T}_{\mu} T_{\mu}\right) \operatorname{tr}\left(\mathcal{W}_{+}\right)
\end{aligned}
$$

where the trace is taken in flavor space, and the notation, (...), denotes contractions of the flavor (tensor) indices as defined in e.g. Ref. [27]. The "twisted mass" spurion field is defined by

$$
\mathcal{M}_{ \pm}^{t w}=\frac{1}{2}\left[\xi^{\dagger} m_{Q}^{t w} \xi^{\dagger} \pm \xi\left(m_{Q}^{t w}\right)^{\dagger} \xi\right], \quad m_{Q}^{t w}=\frac{\chi^{\prime}}{2 B_{0}}
$$

with $m_{Q}^{t w}$ being the "twisted" mass spurion for the baryons. The "Wilson" (discretization) spurion field is defined by

$$
\mathcal{W}_{ \pm}=\frac{1}{2}\left(\xi^{\dagger} w_{Q} \xi^{\dagger} \pm \xi w_{Q}^{\dagger} \xi\right), \quad w_{Q}=\frac{\Lambda_{\mathrm{QCD}}^{2}}{2 W_{0}} \hat{A}
$$

with $w_{Q}$ being the Wilson spurion for the baryons. Note that we have made simplifications using the properties of $S U(2)$ matrices when writing down Eq. (21). When setting the spurions to their constant values, $\mathcal{W}_{+}$is proportional to the identity matrix in flavor space. Thus the operators $\bar{N} \mathcal{W}_{+} N$ and $\left(\bar{T}_{\mu} \mathcal{W}_{+} T_{\mu}\right)$, although allowed under the symmetries of tmLQCD, are not independent operators with respect to $\bar{N} N \operatorname{tr}\left(\mathcal{W}_{+}\right)$and $\left(\bar{T}_{\mu} T_{\mu}\right) \operatorname{tr}\left(\mathcal{W}_{+}\right)$respectively. This is also true of the nucleon and delta operators involving $\mathcal{M}_{+}^{t w}$ in the isospin limit (but not away from it). The independent operators we choose to write down are those with the simplest flavor contractions, and this will be the case henceforth whenever we make simplifications using the properties of $S U(2)$.

In Eq. (21), the four-vector, $v_{\mu}$, is the heavy baryon four-velocity, and our conventional here is that $v \cdot v=1$. The parameter, $\Delta$, is the mass splitting between the nucleons and deltas which is independent of the quark masses (often referred to as the nucleon-delta mass splitting in the chiral limit), and we treat $\Delta \sim m_{\pi} \sim \varepsilon^{2}$ following [24, 25, 26, 28]. The chiral covariant derivative, $D_{\mu}$, acts on the nucleon and delta fields as

$$
\begin{aligned}
\left(D_{\mu} N\right)_{i} & =\partial_{\mu} N_{i}+\left(\mathcal{V}_{\mu}\right)_{i}{ }^{j} N_{j} \\
\left(D_{\mu} T^{\nu}\right)_{i j k} & =\partial_{\mu} T_{i j k}^{\nu}+\left(\mathcal{V}_{\mu}\right)_{i}{ }^{i^{\prime}} T_{i^{\prime} j k}^{\nu}+\left(\mathcal{V}_{\mu}\right)_{j}{ }^{j^{\prime}} T_{i j^{\prime} k}^{\nu}+\left(\mathcal{V}_{\mu}\right)_{k}{ }^{k^{\prime}} T_{i j k^{\prime}}^{\nu}
\end{aligned}
$$

The vector and axial-vector fields are defined by

$$
\mathcal{V}_{\mu}=\frac{1}{2}\left(\xi \partial_{\mu} \xi^{\dagger}+\xi^{\dagger} \partial_{\mu} \xi\right), \quad \mathcal{A}_{\mu}=\frac{i}{2}\left(\xi \partial_{\mu} \xi^{\dagger}-\xi^{\dagger} \partial_{\mu} \xi\right), \quad \xi^{2}=\Sigma
$$

The dimensionless low energy constants (LECs), $\alpha_{M}, \sigma_{M}, \gamma_{M}$, and $\bar{\sigma}_{M}$ have the same numerical values as in the usual untwisted two-flavor $\mathrm{HB} \chi \mathrm{PT}$.

As was noted in Ref. [18], the shifting from $\chi$ to $\chi^{\prime}=\chi+\hat{A}$, which corresponds to the shift of the physical mass $m$ to $m^{\prime}$ at the quark level does not, in general, remove the discretization $(\hat{A})$ term, and this is seen explicitly here with the presence of the discretization terms.

The Lagrangian describing the interactions of the nucleons and deltas with the pions is

$$
\mathcal{L}_{\chi}=2 g_{A} \bar{N} S \cdot A N-2 g_{\Delta \Delta} \bar{T}_{\mu} S \cdot \mathcal{A} T_{\mu}+g_{\Delta N}\left[\bar{T}_{\mu}^{k j i} \mathcal{A}_{i}^{\mu, i^{\prime}} \epsilon_{j i^{\prime}} N_{k}+h . c .\right], \quad i, j, k=1,2,3 .
$$

The tensor $\epsilon_{i j}$ is the rank-2 analogue of the totally antisymmetric tensor $\epsilon_{i j k}$. The vector $S_{\mu}$ is the covariant spin operator [24, 25]. The LECs in (26) are the same as those in the untwisted two-flavor HB $\chi \mathrm{PT}$. Note that the $\mathcal{O}\left(\varepsilon^{2}\right)$ free Lagrangian (21) and interaction Lagrangian (26) are the same as those given in Ref. 21] when the twist is removed, i.e. when $\mu=0$. With non-vanishing twist, the mass operators carry a twisted component and the vacuum is "twisted" from the identity to point in the direction of the twist (the flavor $\tau_{1}$-direction here) [18].

Following Ref. [18], we expand $\Sigma$ about its vacuum expectation value, defining the physical pion fields and the physical $\xi$ fields by

$$
\Sigma=\mathcal{T} \Sigma_{p h} \mathcal{T}, \quad \xi=\mathcal{T} \xi_{p h} V\left(\xi_{p h}\right), \quad \mathcal{T}=\exp \left(i \omega_{0} \tau_{1} / 2\right), \quad \Sigma_{p h}=\exp (i \sqrt{2} \pi \cdot \tau / f), \quad \mathcal{T}, V \in S U(2),
$$

Now, if we make the following chiral transformation (under which the effective chiral Lagrangian is invariant)

$$
\begin{gathered}
\Sigma \rightarrow L \Sigma R^{\dagger}, \quad \xi \rightarrow L \xi V^{\dagger} \equiv V \xi R^{\dagger}, \quad N^{i} \rightarrow V^{i j} N^{j}, \quad T_{\mu}^{i j k} \rightarrow V^{i i^{\prime}} V^{j j^{\prime}} V^{k k^{\prime}} T_{\mu}^{i^{\prime} j^{\prime} k^{\prime}}, \\
\chi^{\prime} \rightarrow L \chi^{\prime} R^{\dagger}, \quad \hat{A} \rightarrow L \hat{A} R^{\dagger}, \quad L, R, V \in S U(2),
\end{gathered}
$$


using the particular $S U(2)$ matrices $L=R^{\dagger}=\mathcal{T}^{\dagger}$, we have in the transformed effective chrial Lagrangian

$$
\Sigma \rightarrow \Sigma_{p h}, \quad \xi \rightarrow \xi_{p h}, \quad \mathcal{A}_{\mu} \rightarrow \frac{i}{2}\left(\xi_{p h} \partial_{\mu} \xi_{p h}^{\dagger}-\xi_{p h}^{\dagger} \partial_{\mu} \xi_{p h}\right), \quad \mathcal{V}_{\mu} \rightarrow \frac{1}{2}\left(\xi_{p h} \partial_{\mu} \xi_{p h}^{\dagger}+\xi_{p h}^{\dagger} \partial_{\mu} \xi_{p h}\right),
$$

and

$$
\begin{array}{rlrl}
\mathcal{M}_{ \pm}^{t w} \rightarrow \mathcal{M}_{ \pm} & =\frac{1}{2}\left(\xi_{p h}^{\dagger} m_{Q} \xi_{p h}^{\dagger} \pm \xi_{p h} m_{Q}^{\dagger} \xi_{p h}\right), & m_{Q}^{t w} \rightarrow m_{Q}=\mathcal{T}^{\dagger} \frac{\chi^{\prime}}{2 B_{0}} \mathcal{T}^{\dagger} \\
\mathcal{W}_{ \pm} \rightarrow \mathcal{W}_{ \pm}^{t w}=\frac{1}{2}\left[\xi_{p h}^{\dagger} w_{Q}^{t w} \xi_{p h}^{\dagger} \pm \xi_{p h}\left(w_{Q}^{t w}\right)^{\dagger} \xi_{p h}\right], & w_{Q} \rightarrow w_{Q}^{t w}=\mathcal{T}^{\dagger}\left(\frac{\Lambda_{Q \mathrm{CD}}^{2}}{2 W_{0}} \hat{A}\right) \mathcal{T}^{\dagger}
\end{array}
$$

Note that since $L=R^{\dagger}=\mathcal{T}^{\dagger} \in S U(2)$, and $\xi=L^{\dagger} \xi_{p h} V \equiv V^{\dagger} \xi_{p h} R$,

$$
\Sigma=L^{\dagger} \Sigma_{p h} R=\xi^{2}=\left(L^{\dagger} \xi_{p h} V\right) \cdot\left(V^{\dagger} \xi_{p h} R\right)=L^{\dagger} \xi_{p h}^{2} R \Longrightarrow \xi_{p h}^{2}=\Sigma_{p h} .
$$

We see that the $\xi$ field is now $\xi_{p h}$, the field associated with the physical pions, and the twist is transferred from the twisted mass $\left(\mathcal{M}_{ \pm}^{t w}\right)$ term to the "twisted Wilson" $\left(\mathcal{W}_{ \pm}^{t w}\right)$ term, making the mass term in the $\mathrm{HB} \chi \mathrm{PT}$ now the same as that in the untwisted theory. The new mass spurion, $m_{Q}$, and the "twisted Wilson" spurion, $w_{Q}^{t w}$, now take constant values

$$
m_{Q} \longrightarrow m_{q}-\epsilon_{q} \tau_{3}, \quad w_{Q}^{t w} \longrightarrow a \Lambda_{Q C D}^{2} \exp \left(-i \omega_{0} \tau_{1}\right) .
$$

We will call this the "physical pion basis" since this is the basis where the pions are physical [18], and we will work in this basis from now on, unless otherwise specified. ${ }^{4}$ A technical point we note here is that, in the isospin limit where twisting can be implemented by any of the three Pauli matrices, say $\tau_{k}$, the physical pion basis can be found following the same recipe detailed above but with $\tau_{1}$ in $\mathcal{T}$ replaced by $\tau_{k}$ throughout.

Rotating to the physical pion basis where the $\xi$ field is now the physical $\xi_{p h}$ field in all field quantities, the form of the interaction Lagrangian remains unchanged as given in (26), while the $\mathcal{O}\left(\varepsilon^{2}\right)$ free heavy baryon chiral Lagrangian (21) changes to

$$
\begin{aligned}
\mathcal{L}_{\chi}= & \bar{N} i v \cdot D N-2 \alpha_{M} \bar{N} \mathcal{M}_{+} N-2 \sigma_{M} \bar{N} N \operatorname{tr}\left(\mathcal{M}_{+}\right)-2 \sigma_{W} \bar{N} N \operatorname{tr}\left(\mathcal{W}_{+}^{t w}\right) \\
& +\left(\bar{T}_{\mu} i v \cdot D T_{\mu}\right)+\Delta\left(\bar{T}_{\mu} T_{\mu}\right)+2 \gamma_{M}\left(\bar{T}_{\mu} \mathcal{M}_{+} T_{\mu}\right)-2 \bar{\sigma}_{M}\left(\bar{T}_{\mu} T_{\mu}\right) \operatorname{tr}\left(\mathcal{M}_{+}\right)-2 \bar{\sigma}_{W}\left(\bar{T}_{\mu} T_{\mu}\right) \operatorname{tr}\left(\mathcal{W}_{+}^{t w}\right) .
\end{aligned}
$$

Note that $\mathcal{W}_{+}^{t w}$ is also proportional to the identity matrix in flavor space when set to its constant value. Thus if we build the free chiral Lagrangian directly in the physical pion basis, the same simplifications due to $S U(2)$ we used in writing down Eq. (21) apply. Note that at this point, one can not yet tell whether the nucleon $(N)$ and the delta $\left(T_{\mu}\right)$ fields are physical. This has to be determined by the theory itself. We will return to this point when calculating the nucleon and delta masses below.

At $\mathcal{O}\left(\varepsilon^{4}\right)$, there are contributions from $\mathcal{O}(a \mathrm{~m})$ and $\mathcal{O}\left(a^{2}\right)$ operators. The enumeration of the operators is similar to that set out in Ref. [21], except now the Wilson spurion field carries a twisted component. The operators appearing in the $\mathcal{O}\left(\varepsilon^{4}\right)$ chiral Lagrangian will involve two insertions off the following: $\mathcal{M}_{ \pm}, \mathcal{W}_{ \pm}^{t w}$, and the axial current $\mathcal{A}_{\mu}$. Note that since parity combined with flavor is conserved in tmLQCD, any one insertion of $\mathcal{M}_{-}$or $\mathcal{W}_{-}^{t w}$ must be accompanied by another insertion of $\mathcal{M}_{-}$or $\mathcal{W}_{-}^{t w}$. Now operators with two insertions of $\mathcal{M}_{+}$or $\mathcal{A}_{\mu}$, which contribute to baryon masses at tree and one-loop level respectively, have the same form as those in the untwisted theory (and so give the same contribution). These have been written down in 29] and will not be repeated here. Operators with an insertion of either a combination of $v \cdot \mathcal{A}$ and $\mathcal{M}_{-}$(which have the same form as in the untwisted theory), or a combination of $v \cdot \mathcal{A}$ and $\mathcal{W}_{-}^{t w}$, will also not contribute to the baryon masses at $\mathcal{O}\left(\varepsilon^{4}\right)$.

At $\mathcal{O}(a \mathrm{~m})$, there are two independent operators contributing to the masses in the nucleon sector

$$
\mathcal{L}_{\chi}=-\frac{1}{\Lambda_{\chi}}\left[n_{1}^{W M_{+}} \bar{N} \mathcal{M}_{+} N \operatorname{tr}\left(\mathcal{W}_{+}^{t w}\right)+n_{2}^{W M_{+}} \bar{N} N \operatorname{tr}\left(\mathcal{M}_{+}\right) \operatorname{tr}\left(\mathcal{W}_{+}^{t w}\right)\right]
$$

\footnotetext{
4 As detailed in Ref. [18], the twist angle that one determines non-perturbatively in practice, call it $\omega$, will differ from $\omega_{0}$ by $O(a)$. This will give rise to a relative $O(a)$ contribution to the pion terms. But since the pions come into baryon calculations only through loops, the correction will be of higher order than we work. Thus to the accuracy we work, we may use either $\omega$ or $\omega_{0}$.
} 
and two independent operators contributing to the masses in the delta sector

$$
\mathcal{L}_{\chi}=\frac{1}{\Lambda_{\chi}}\left[t_{1}^{W M_{+}}\left(\bar{T}_{\mu} \mathcal{M}_{+} T_{\mu}\right) \operatorname{tr}\left(\mathcal{W}_{+}^{t w}\right)+t_{2}^{W M_{+}}\left(\bar{T}_{\mu} T_{\mu}\right) \operatorname{tr}\left(\mathcal{W}_{+}^{t w}\right) \operatorname{tr}\left(\mathcal{M}_{+}\right)\right]
$$

where $\Lambda_{\chi} \equiv 4 \pi f{ }^{5}$. Note that there are no operators involving the commutator, $\left[\mathcal{M}_{+}, \mathcal{W}_{+}^{t m}\right]$, because it is identically zero. There are also operators involving $\mathcal{M}_{-} \otimes \mathcal{W}_{-}^{t w}$ at $\mathcal{O}(a \mathrm{~m})$, but these again do not contribute to the baryon masses at the order we work.

Note that the scale used in Eqs. (34) and (35) above to make the dimensions correct is not the QCD scale, $\Lambda_{\mathrm{QCD}}$, but the $\chi \mathrm{PT}$ scale, $\Lambda_{\chi}$. This follows from the naive dimensional analysis (NDA) of Ref. [30], and from the fact that these operators also function as counter terms for divergences arising from the leading loop diagrams, which have contributions proportional to $\mathcal{O}(a \mathrm{~m})$. We use the same analysis to set the dimensionfull scale in all the following operators which contribute to the nucleon and delta masses to the order we work.

At $\mathcal{O}\left(a^{2}\right)$, there are operators that do not break the chiral symmetry arising from the bilinear operators and fourquark operators (see e.g. Ref. [13] for a complete listing) in the $\mathcal{O}\left(a^{2}\right)$ part of $\mathcal{L}_{\text {eff }}$. These give rise to the tmHB $\chi \mathrm{PT}$ operators

$$
\mathcal{L}_{\chi}=a^{2} \Lambda_{\mathrm{QCD}}^{3}\left[-b \bar{N} N+t\left(\bar{T}_{\mu} T_{\mu}\right)\right] .
$$

There are also chiral symmetry preserving but $O(4)$ rotation symmetry breaking operators which arise from the bilinear operator of the form $a^{2} \bar{\psi} \gamma_{\mu} D_{\mu} D_{\mu} D_{\mu} \psi$ in the $\mathcal{O}\left(a^{2}\right)$ part of $\mathcal{L}_{\text {eff }}$. These give rise to the tmHB $\chi \mathrm{PT}$ operators

$$
\mathcal{L}_{\chi}=a^{2} \Lambda_{\mathrm{QCD}}^{3}\left[-b_{v} \bar{N} v_{\mu} v_{\mu} v_{\mu} v_{\mu} N+t_{v}\left(\bar{T}_{\nu} v_{\mu} v_{\mu} v_{\mu} v_{\mu} T_{\nu}\right)+t_{\bar{v}}\left(\bar{T}_{\mu} v_{\mu} v_{\mu} T_{\mu}\right)\right] .
$$

Note that these chiral symmetry preserving operators are clearly not affected by twisting (the $O(4)$ symmetry breaking operator at the quark level from which they arise involve only derivatives with no flavor structure, and $\left.\left\{\gamma_{\mu}, \gamma_{5}\right\}=0\right)$, and so they have the same form and contribute to the baryon masses in the same way as in the untwisted theory. The chiral symmetry breaking operators at $\mathcal{O}\left(a^{2}\right)$ are those with two insertions of the Wilson spurion fields. For the nucleons, there are two such independent operators

$$
\mathcal{L}_{\chi}=-\frac{1}{\Lambda_{\mathrm{QCD}}}\left[n_{1}^{W_{+}} \bar{N} N \operatorname{tr}\left(\mathcal{W}_{+}^{t w}\right) \operatorname{tr}\left(\mathcal{W}_{+}^{t w}\right)+n_{1}^{W_{-}} \bar{N} N \operatorname{tr}\left(\mathcal{W}_{-}^{t w} \mathcal{W}_{-}^{t w}\right)\right],
$$

and for the deltas, there are three such independent operators

$$
\mathcal{L}_{\chi}=\frac{1}{\Lambda_{\mathrm{QCD}}}\left[t_{1}^{W_{+}}\left(\bar{T}_{\mu} T_{\mu}\right) \operatorname{tr}\left(\mathcal{W}_{+}^{t w}\right) \operatorname{tr}\left(\mathcal{W}_{+}^{t w}\right)+t_{1}^{W_{-}}\left(\bar{T}_{\mu} T_{\mu}\right) \operatorname{tr}\left(\mathcal{W}_{-}^{t w} \mathcal{W}_{-}^{t w}\right)+t_{2}^{W_{-}} \bar{T}_{\mu}^{k j i}\left(\mathcal{W}_{-}^{t w}\right)^{i i^{\prime}}\left(\mathcal{W}_{-}^{t w}\right)^{j j^{\prime}} T_{\mu}^{i^{\prime} j^{\prime} k}\right] .
$$

In the isospin limit where the mass splitting vanishes $\left(\epsilon_{q} \rightarrow 0\right)$, more simplifications occur in the $\mathcal{O}\left(\varepsilon^{4}\right)$ chiral Lagrangian. The nucleon operators with coefficients $n_{1}^{W M_{+}}$and $n_{2}^{W M_{+}}$are the same up to a numerical factor, and the same holds for delta operators with coefficients $t_{1}^{W M_{+}}, t_{2}^{W M_{+}}, t_{3}^{W M_{+}}$, and for operators with coefficient $t_{1}^{W-}$ and $t_{2}^{W_{-}}$.

Note that in the untwisted limit, the $\mathcal{O}\left(\varepsilon^{4}\right)$ chiral Lagrangian reduces to that given in Ref. 21]. In particular, with the twist set to zero, operators with two insertions of $\mathcal{W}_{-}^{t w}$ will not contribute to the nucleon or the delta mass until $\mathcal{O}\left(a^{2} \mathrm{~m}\right) \sim \mathcal{O}\left(\varepsilon^{6}\right)$. But for non-vanishing twist, they contribute at $\mathcal{O}\left(a^{2}\right)$.

\section{NUCLEON AND DELTA MASSES IN THE ISOSPIN LIMIT}

There have been extensive studies of the nucleon masses, and to a lesser extent, the delta masses in $\mathrm{HB} \chi \mathrm{PT}$ and other variants of $\chi \mathrm{PT}$. A partial list of references of these studies includes 21, 26, 28, 29, 31, 32, 33, 34, 35, 36, 37, 38, 39, 40, 41]. In this work, we are concerned with corrections to the masses of the nucleons and the deltas due to the effect of the twisted mass parameter. We will therefore only give expressions for the mass corrections arising

\footnotetext{
${ }^{5}$ We will use this definition as our convention, which differs from the more standard convention, $\Lambda_{\chi}=2 \sqrt{2} \pi f$, employed by other authors.
} 

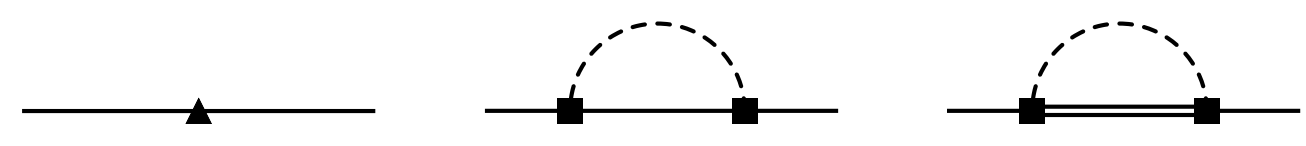

FIG. 1: Diagrams depicting LO and NLO mass contributions to the nucleons in tmHB $\chi \mathrm{PT}$ in the physical pion basis. The solid, double solid and dashed lines denote nucleons, deltas and pions respectively. The solid triangle represents an insertion of the twisted Wilson operator as given in Eq. (33). The solid squares denote the couplings of the baryons to the axial current whose form is given in Eq. (26).

from the effects of lattice discretization and twisting in tmLQCD. A calculation of the nucleon and delta masses in the continuum in infinite volume to $\mathcal{O}\left(m_{q}^{2}\right)$ can be found in Ref. [29]. The mass corrections due to finite lattice spacing to $\mathcal{O}\left(a^{2}\right)$ in the untwisted theory with Wilson quarks can be found in Ref. [21], and the leading finite volume modifications to the nucleon mass can be found in Ref. [42].

In this section, we present the results of nucleon and delta masses calculated in tmHB $\chi \mathrm{PT}$, in the isospin limit, where the quark doublet is mass-degenerate, and the twist is implemented by $\tau_{3}$. As we discussed in Sec. the isospin limit, the content of tmLQCD is the same regardless of which Pauli matrix is used to implement the twist - the action for one choice is related to another by a flavor-vector rotation. This must also hold true of the effective chiral theory that arises from tmLQCD. Indeed, the heavy baryon Lagrangian constructed in Sec. [C] with $\tau_{1}$-twisting can be rotated into that with $\tau_{3}$-twisting by making a vector transformation, which is given by Eq. (28) but with $L=R=V=\exp \left(i \frac{\pi}{4} \tau_{2}\right)$.

\section{A. Nucleon Masses in the Isospin Limit}

In the continuum, the mass of the nucleons in infinite volume $\mathrm{HB} \chi \mathrm{PT}$ with two flavor-degenerate quarks are organized as an expansion in powers of the quark mass, which can be written as ${ }^{6}$

$$
M_{N_{i}}=M_{0}\left(\Delta, \Lambda_{R}\right)+M_{N_{i}}^{(1)}\left(\Delta, \Lambda_{R}\right)+M_{N_{i}}^{(3 / 2)}\left(\Delta, \Lambda_{R}\right)+M_{N_{i}}^{(2)}\left(\Delta, \Lambda_{R}\right)+\ldots
$$

where $N_{i}$ stands for either the proton $(i=p)$ or the neutron $(i=n)$, and $M_{N_{i}}^{(n)}$ is the contribution to the $i^{t h}$ nucleon of $\mathcal{O}\left(m_{q}^{n}\right)$ calculated in the continuum and infinite volume two-flavor $\chi \mathrm{PT}$ in the isospin limit [29]. The quantity, $M_{0}\left(\Delta, \Lambda_{R}\right)$, is the renormalized nucleon mass in the chiral limit; it is independent of $m_{q}$ and $N_{i}$, but depends the renormalization scale, $\Lambda_{R}$, and on $\Delta$, the renormalized mass splitting between the nucleons and deltas which is independent of the quark masses. Here, we are interested in the corrections to this formula due to the effects of lattice discretization and twisting arising from tmLQCD. We denote these lattice corrections to the nucleon mass at $\mathcal{O}\left(\varepsilon^{2 n}\right) \sim \mathcal{O}\left(m_{q}^{n}\right) \sim \mathcal{O}\left(a^{n}\right)$ (factors of $\Lambda_{\mathrm{QCD}}$ needed to make the dimensions correct are implicit here) as $\delta M_{N_{i}}^{(n)}$, and the nucleon mass in tmHB $\chi \mathrm{PT}$ is now written as

$$
M_{N_{i}}^{t m}=M_{0}+\left(M_{N_{i}}^{(1)}+\delta M_{N_{i}}^{(1)}\right)+\ldots
$$

Throughout this work, we use dimensional regularization with a modified minimal subtraction scheme where we consistently subtract off terms proportional to

$$
\frac{1}{\varepsilon}-\gamma_{E}+1+\log 4 \pi
$$

The leading correction in $\operatorname{tm} \chi \mathrm{PT}$ comes in at tree level, arising from the twisted Wilson nucleon operator in the free heavy baryon Lagrangian (33). It reads

$$
\delta M_{N_{i}}^{(1)}(\omega)=-4 \sigma_{W} a \Lambda_{\mathrm{QCD}}^{2} \cos (\omega),
$$

where to the accuracy we work, $\omega$ can either be $\omega_{0}$ or the twist angle non-perturbatively determined. Note that this correction is the same for both the proton and the neutron. At leading order, the nucleon mass is automatically $\mathcal{O}(a)$ improved, as $\delta M_{N_{i}}^{(1)}$ vanishes at maximal twist, $\omega=\pi / 2$. At zero twist, $\omega=0$, it reduces to that in the untwisted theory [20, 21].

\footnotetext{
${ }^{6}$ Here we use a different convention from some of the more recent nucleon mass calculations.
} 

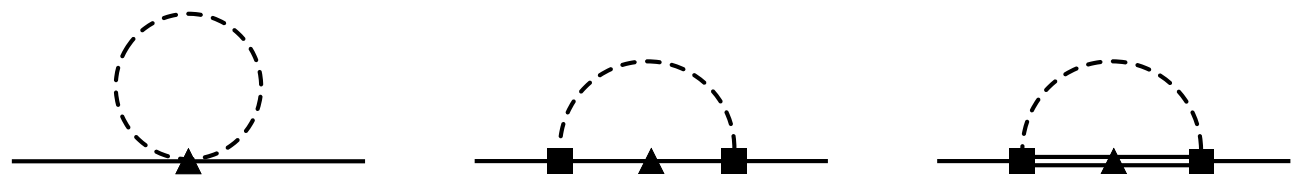

FIG. 2: Diagrams depicting mass contributions to the nucleons at next-to-next-to-leading order (NNLO) in tmHB $\chi \mathrm{PT}$ in the physical pion basis. The solid, double solid and dashed lines denote nucleons, deltas and pions respectively. The solid triangle denotes an insertion of the twisted Wilson operator as given in (33). The solid squares denote the coupling of the baryons to the axial current whose form is given in (26). The clear triangle denotes a tree level insertion of the operators given in Eqs. (34) and (36) - (38).

The next contribution to the nucleon mass comes from the leading pion loop diagrams shown in Fig. 11 However, at the order we work, the form of the $\mathcal{O}\left(\mathrm{m}^{3 / 2}\right)$ nucleon mass contribution is unchanged from the continuum. For completeness we give its full expression here

$$
M_{N_{i}}^{(3 / 2)}=-\frac{3}{16 \pi f^{2}} g_{A}^{2} m_{\pi}^{3}-\frac{8 g_{\Delta N}^{2}}{3(4 \pi f)^{2}} \mathcal{F}\left(m_{\pi}, \Delta, \Lambda_{R}\right),
$$

where $m_{\pi}^{2}=M^{\prime}=2 B_{0} m_{q}$, is the physical pion mass-squared given in Eq. (16), and the function $\mathcal{F}$ is given by

$$
\mathcal{F}\left(m, \delta, \Lambda_{R}\right)=\left(m^{2}-\delta^{2}\right)\left[\sqrt{\delta^{2}-m^{2}} \log \left(\frac{\delta-\sqrt{\delta^{2}-m^{2}+i \varepsilon}}{\delta+\sqrt{\delta^{2}-m^{2}+i \varepsilon}}\right)-\delta \log \left(\frac{m^{2}}{\Lambda_{R}^{2}}\right)\right]-\frac{\delta}{2} m^{2} \log \left(\frac{m^{2}}{\Lambda_{R}^{2}}\right) .
$$

The corrections to $M_{N_{i}}^{(2)}$ come from both the tree level and the one-loop diagrams, as shown in Fig. [2. The twisted Wilson operator in the free Lagrangian (33) gives rise to a tadpole diagram, which produces a contribution of $\mathcal{O}(a \mathrm{~m})$. The leading Wilson spurions also contribute to $\mathcal{O}(a \mathrm{~m})$ when inserted inside the pion-nucleon loops, and are partly cancelled by wavefunction corrections. The tree level contributions come from the operators given in Eqs. (34) and (36) - (38). Just as in the untwisted continuum theory, these act both as the higher dimensional operators and as counter terms that renormalize divergences from the lower order loop contributions. For instance, coefficients $n_{1}^{W M_{+}}$ and $n_{2}^{W M_{+}}$are renormalized to absorb divergences from the tadpole and one-loop contributions mentioned above. As mentioned in Sec. [I] the operators with these coefficients are suppressed by $\Lambda_{\chi}$ instead of $\Lambda_{\mathrm{QCD}}$ because they are the counterterms for the loop divergences. These coefficients are taken to be the renormalized coefficients (finite) in the mass calculations, and to contain the counter terms needed in our renormalization scheme. The corrections to $M_{N_{i}}^{(2)}$ read

$$
\begin{aligned}
\delta M_{N_{i}}^{(2)}(\omega)= & 12 \sigma_{W} \frac{a \Lambda_{\mathrm{QCD}}^{2}}{\Lambda_{\chi}^{2}} m_{\pi}^{2} \log \left(\frac{m_{\pi}^{2}}{\Lambda_{R}^{2}}\right) \cos (\omega)+16 g_{\Delta N}^{2}\left(\bar{\sigma}_{W}-\sigma_{W}\right) \frac{a \Lambda_{\mathrm{QCD}}^{2}}{\Lambda_{\chi}^{2}}\left[\mathcal{J}\left(m_{\pi}, \Delta, \Lambda_{R}\right)+m_{\pi}^{2}\right] \cos (\omega) \\
& -2\left(n_{1}^{W M_{+}}+2 n_{2}^{W M_{+}}\right) \frac{a \Lambda_{\mathrm{QCD}}^{2}}{\Lambda_{\chi}} m_{q} \cos (\omega)-a^{2} \Lambda_{\mathrm{QCD}}^{3}\left(b+b_{v}\right) \\
& +a^{2} \Lambda_{\mathrm{QCD}}^{3}\left(2 n_{1}^{W_{-}} \sin ^{2}(\omega)-4 n_{1}^{W_{+}} \cos ^{2}(\omega)\right),
\end{aligned}
$$

where the function $\mathcal{J}$ is given by

$$
\mathcal{J}\left(m, \delta, \Lambda_{R}\right)=\left(m^{2}-2 \delta^{2}\right) \log \left(\frac{m^{2}}{\Lambda_{R}^{2}}\right)+2 \delta \sqrt{\delta^{2}-m^{2}} \log \left(\frac{\delta-\sqrt{\delta^{2}-m^{2}+i \varepsilon}}{\delta+\sqrt{\delta^{2}-m^{2}+i \varepsilon}}\right) .
$$

Note that the $\mathcal{O}\left(\varepsilon^{4}\right)$ corrections are again the same for both the proton and the neutron. At maximal twist, the $\mathcal{O}\left(\varepsilon^{4}\right)$ corrections are given by

$$
\delta M_{N_{i}}^{(2)}(\omega=\pi / 2)=a^{2} \Lambda_{\mathrm{QCD}}^{3}\left(2 n_{1}^{W_{-}}-b-b_{v}\right),
$$

while at zero twist, these reduces to the corrections given in Ref. 21]. We see that the nucleon masses are also automatically $\mathcal{O}(a)$ improved at $\mathcal{O}\left(\varepsilon^{4}\right)$.

To the order we work, the expressions for the nucleon mass corrections in tmHB $\chi \mathrm{PT}$ given in Eqs. (43) and (46), together with the untwisted continuum $\mathrm{HB} \chi \mathrm{PT}$ expressions for the nucleon masses, provide the functional form for the dependence of the nucleon masses on the twist and angle, $\omega$, and the quark mass, $m_{q}$, which can be used to fit the lattice data. 

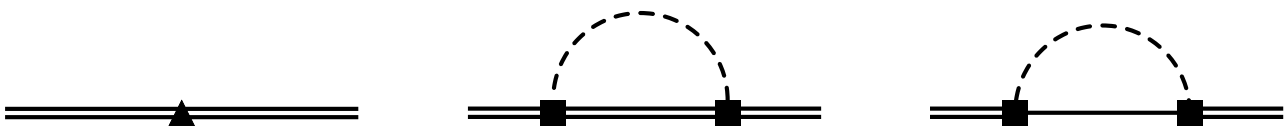

FIG. 3: Diagrams depicting LO and NLO mass contributions to the deltas in tmHB $\chi \mathrm{PT}$ in the physical pion basis. The solid, double solid and dashed lines denote nucleons, deltas and pions respectively. The solid triangle is an insertion of the twisted Wilson operator given in Eq. [33). The solid squares denote the couplings of the baryons to the axial current whose form is given in Eq. (26).

\section{B. Delta Masses in the Isospin Limit}

Before we present our delta mass expressions, we stress that they can only be fit to tmLQCD data for sufficiently large quark masses such that the delta is a stable particle. This corresponds to $m_{\pi} \gtrsim 300 \mathrm{MeV}$, which is pushing the bounds of validity of chiral perturbation theory [42]. However, these expressions can be used to study the convergence of $\chi \mathrm{PT}$ for these pion masses, where the LECs can be determined. With the value of the LECs known, the mass calculations can be analytically continued to pion masses where the delta becomes unstable, and be used to predict e.g. their lifetimes.

The delta masses in the continuum, infinite volume $\mathrm{HB} \chi \mathrm{PT}$ with a flavor doublet of degenerate quarks have a similar expansion as that for the nucleons given in Eq. (40). The mass expansion of the $i^{\text {th }}$ delta is conventionally written

$$
M_{T_{i}}=M_{0}\left(\Delta, \Lambda_{R}\right)+\Delta+M_{T_{i}}^{(1)}\left(\Delta, \Lambda_{R}\right)+M_{T_{i}}^{(3 / 2)}\left(\Delta, \Lambda_{R}\right)+M_{T_{i}}^{(2)}\left(\Delta, \Lambda_{R}\right)+\ldots
$$

where

$$
T_{1}=\Delta^{++}, \quad T_{2}=\Delta^{+}, \quad T_{3}=\Delta^{0}, \quad T_{4}=\Delta^{-},
$$

and $M_{T_{i}}^{(n)}$ is the contribution to the $i^{t h}$ delta of $\mathcal{O}\left(m_{q}^{n}\right)$ calculated in the continuum and infinite volume two-flavor $\chi \mathrm{PT}$ in the isospin limit [29]. As in the case of the nucleons, $M_{0}\left(\Delta, \Lambda_{R}\right)$ is the renormalized nucleon mass in the chiral limit, and the parameter, $\Delta$, is the renormalized mass splitting between the nucleons and deltas which is independent of the quark masses. ${ }^{7}$

Both parameters, $M_{0}$ and $\Delta$, are flavor singlets, and are therefore renormalized in the same way. In the nucleon sector, all flavor singlet mass contributions that are independent of the quark mass go into renormalizing the parameter $M_{0}$. In the delta sector, the choice of which parameter to renormalize, $M_{0}$ or $\Delta$, is arbitrary. For convenience we choose $M_{0}$ to be the renormalized nucleon mass in the chiral limit. Thus, $\Delta$ is simply the difference between the nucleon and delta masses in the chiral limit, to a given order in the chiral expansion. Both $M_{0}$ and $\Delta$ are parameters which must be fit to the lattice data. For the delta masses, we use the same renormalization scheme as for the nucleons.

We again denote the correction of $\mathcal{O}\left(\varepsilon^{2 n}\right)$ to the delta masses due to the effects of lattice discretization and twisting arising from tmLQCD as $\delta M_{T_{i}}^{(n)}$, and the delta masses in tmHB $\chi \mathrm{PT}$ are written as

$$
M_{T_{i}}^{t m}=M_{0}+\Delta+\left(M_{T_{i}}^{(1)}+\delta M_{T_{i}}^{(1)}\right)+\ldots
$$

The leading mass correction arises at tree level from the twisted Wilson delta operator given in Eq. (33),

$$
\delta M_{T_{i}}^{(1)}(\omega)=-4 \bar{\sigma}_{W} a \Lambda_{\mathrm{QCD}}^{2} \cos (\omega) .
$$

Just as for the nucleons, this does not split the delta masses and vanishes at maximal twist.

The $\mathcal{O}\left(\varepsilon^{3}\right)$ delta mass contributions are similarly given as for the nucleons. The contributing diagrams are shown in Figure 3. They do not cause any splitting between the deltas, and receive no discretization corrections. For

\footnotetext{
7 There are some subtle issues involved in calculating $\Delta$ in $\mathrm{HB} \chi \mathrm{PT}$ due to the fact that it is a flavor singlet, and so can modify all operators/LECs in the chiral Lagrangian. However, since one can not vary $\Delta$, all the LECs associated with modifying $\Delta$ are not determinable. Nevertheless, one can simply fit for the physical value of $\Delta$ determined by the lattice, and use that value in all expressions in which it arises. For further discussion on this point, see Refs. 29, 40].
} 

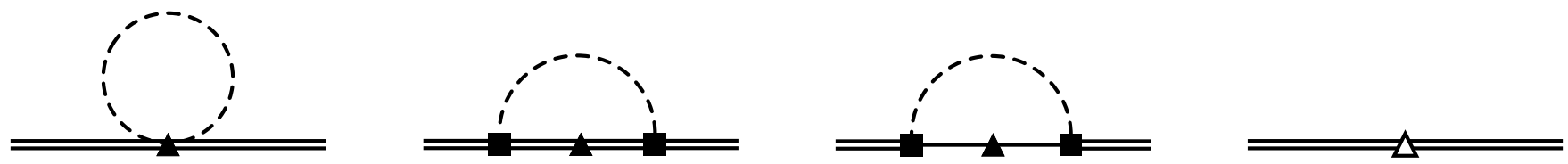

FIG. 4: Diagrams depicting mass contributions to the deltas at NNLO in tmHB $\chi \mathrm{PT}$ in the physical pion basis. The solid, double solid and dashed lines denote nucleons, deltas and pions respectively. The solid triangle denotes an insertion of the discretization operator in Eq. (33). The solid squares denote the coupling of the baryons to the axial current whose form is given in Eq. (26). The clear triangle denotes a tree level insertion of the operators given in Eqs. (35) - (37) and (39).

completeness, we list the full mass expression at this order here

$$
M_{T_{i}}^{(3 / 2)}=-\frac{25 g_{\Delta \Delta}^{2}}{432 \pi f^{2}} m_{\pi}^{3}-\frac{2 g_{\Delta N}^{2}}{3(4 \pi f)^{2}} \mathcal{F}\left(m_{\pi},-\Delta, \Lambda_{R}\right) .
$$

For $m_{\pi}>\Delta$, the deltas are stable particles, but for $m_{\pi}<\Delta$, the deltas become unstable (as can be seen from the fact that the function, $\mathcal{F}\left(m_{\pi},-\Delta, \Lambda_{R}\right)$, picks up an imaginary component). When the deltas are unstable, one will not be able to use these expressions to fit the lattice data. However, these expressions can be used fit the lattice data for $m_{\pi} \gtrsim 300 \mathrm{MeV}$, and then analytically continued to light enough pion masses for which the deltas are unstable.

At $\mathcal{O}\left(\varepsilon^{4}\right)$, contributions due to the effects of twisting arise from similar diagrams as in the nucleon case, and are shown in Fig. (4 A splitting in the delta masses first arises at this order, which comes from the operator with coefficient $t_{2}^{W_{-}}$given in (39). The mass corrections read

$$
\begin{aligned}
\delta M_{T_{i}}^{(2)}(\omega)= & 12 \bar{\sigma}_{W} \frac{a \Lambda_{\mathrm{QCD}}^{2}}{\Lambda_{\chi}^{2}} m_{\pi}^{2} \log \left(\frac{m_{\pi}^{2}}{\Lambda_{R}^{2}}\right) \cos (\omega)+4 g_{\Delta N}^{2}\left(\bar{\sigma}_{W}-\sigma_{W}\right) \frac{a \Lambda_{\mathrm{QCD}}^{2}}{\Lambda_{\chi}^{2}} \mathcal{J}\left(m_{\pi},-\Delta, \Lambda_{R}\right) \cos (\omega) \\
& +2\left(t_{1}^{W M_{+}}+2 t_{2}^{W M_{+}}\right) \frac{a \Lambda_{\mathrm{QCD}}^{2}}{\Lambda_{\chi}} m_{q} \cos (\omega)+a^{2} \Lambda_{\mathrm{QCD}}^{3}\left(t+t_{v}\right) \\
& +a^{2} \Lambda_{\mathrm{QCD}}^{3}\left(4 t_{1}^{W_{+}} \cos ^{2}(\omega)-2 t_{1}^{W-} \sin ^{2}(\omega)+t_{2}^{W-} \delta_{T_{i}} \sin ^{2}(\omega)\right)
\end{aligned}
$$

where

$$
\delta_{T_{i}}=\left\{\begin{aligned}
-1 & \text { for } T_{i}=\Delta^{++}, \Delta^{-} \\
\frac{1}{3} & \text { for } T_{i}=\Delta^{+}, \Delta^{0}
\end{aligned}\right.
$$

Note the appearance of the mass splitting, $\delta_{T_{i}}$, in $\delta M_{T_{i}}^{(2)}$. We see from above that starting at $\mathcal{O}\left(\varepsilon^{4}\right)$, the delta multiplet is split into two mass-degenerate pairs, with one pair containing $\Delta^{++}$and $\Delta^{-}$, and the other, $\Delta^{+}$and $\Delta^{0}$. At maximal twist, $\delta M_{T_{i}}^{(2)}$ becomes

$$
\delta M_{T_{i}}^{(2)}(\omega=\pi / 2)=a^{2} \Lambda_{\mathrm{QCD}}^{3}\left(t+t_{v}-2 t_{1}^{W_{-}}+t_{2}^{W_{-}} \delta_{T_{i}}\right),
$$

while at zero twist, it reduces to that given in Ref. [21]. Just as in the nucleon case, the delta masses to $\mathcal{O}\left(\varepsilon^{4}\right)$ are also automatically $\mathcal{O}(a)$ improved.

As is the case with the nucleons, to the order we work, the expressions for the delta mass corrections in tmHB $\chi \mathrm{PT}$ given in Eqs. (52) and (54), together with the untwisted continuum $\mathrm{HB} \chi \mathrm{PT}$ expressions for the delta masses, provide the functional form for the dependence of the delta masses on the twist angle, $\omega$, and the quark mass, $m_{q}$, which can be used to fit the lattice data.

\section{Mass splittings}

Having derived the expressions for the nucleon and delta masses in tmHB $\chi \mathrm{PT}$ to order $\mathcal{O}\left(\varepsilon^{4}\right)$ in the isospin limit, we now focus on the mass splittings between the nucleons and between the deltas. The mass contributions in the continuum, $M_{N_{i}}^{(n)}$ and $M_{T_{i}}^{(n)}$, clearly do not give rise to mass splittings for the nucleons and deltas, since they are 
calculated with degenerate quarks. Therefore, any mass splitting can only come from the mass corrections arising from tmLQCD.

From the results of Sec. IIA and Sec. IIIB we find that to $\mathcal{O}\left(\varepsilon^{4}\right)$, the protons and neutrons remain degenerate, while the delta multiplet splits into two degenerate pairs, with $\Delta^{++}$and $\Delta^{-}$in one pair, and $\Delta^{+}$and $\Delta^{0}$ in the other. The splitting between the degenerate pairs in the delta multiplet is given by

$$
M_{\Delta^{+, 0}}-M_{\Delta^{++,-}}=\frac{4}{3} t_{2}^{W_{-}} a^{2} \Lambda_{\mathrm{QCD}}^{3} \sin ^{2}(\omega)=\frac{4}{3} t_{2}^{W_{-}} a^{2} \Lambda_{\mathrm{QCD}}^{3} \frac{\mu^{2}}{m_{q}^{2}} .
$$

We reiterate here that the $\mathcal{O}(a)$ uncertainty inherent in the definition of the twist angle results in a correction to $M_{\Delta^{+, 0}}-M_{\Delta^{++,-}}$of $\mathcal{O}\left(a^{3}\right) \sim \mathcal{O}\left(\varepsilon^{6}\right)$, which is of higher order than we work. Hence to the accuracy we work, we may use $\omega_{0}$ or any other non-perturbatively determined twist angle for $\omega$ above.

Just as the case of the pion mass splitting worked out in Ref. [18], this delta splitting must vanish quadratically in $a \mu=a m_{q} \sin (\omega)$ on general grounds, since the masses do not violate parity. One would therefore expect, naively, the splitting to be $\mathcal{O}\left(a^{2} m_{q}^{2}\right) \sim \mathcal{O}\left(\varepsilon^{8}\right)$. But as our results show, there is, in fact, a mass dependence in the denominator such that the effect is $\mathcal{O}\left(\varepsilon^{4}\right)$. Suppose we take $a^{-1}=2 \mathrm{GeV}$ and $\Lambda_{\mathrm{QCD}}=0.5 \mathrm{GeV}$, then we would find a mass splitting

$$
M_{\Delta^{+, 0}}-M_{\Delta^{++,-}}=0.04 t_{2}^{W_{-}} \mathrm{GeV}
$$

Using naive dimensional analysis, we expect $t_{2}^{W_{-}} \sim \mathcal{O}(1)$, and we would have a mass splitting of the delta pairs on the order of $50 \mathrm{MeV}$. We point out that in the recent quenched study [19], a mass splitting of on the order of 50 to $100 \mathrm{MeV}$ is found. However, our formula can only be applied to lattice data from unquenched simulations, which have yet to be done, since it is derived in a fully unquenched theory. Nevertheless, it is encouraging that the quenched results are not dramatically different from the estimate we give above with a reasonable value of $\Lambda_{\mathrm{QCD}}$.

Now, the degeneracies we found for the nucleons and the delta multiplet above hold not only at $\mathcal{O}\left(\varepsilon^{4}\right)$, but in fact they hold to all orders in tm $\chi \mathrm{PT}$. This can be understood by considering the lattice Wilson-Dirac operator associated with the action of tmLQCD given in Eq. (2) in the isospin limit with $\tau_{3}$-twisting

$$
D_{W D}=\frac{1}{2} \sum_{\mu} \gamma_{\mu}\left(\nabla_{\mu}^{\star}+\nabla_{\mu}\right)-\frac{r}{2} \sum_{\mu} \nabla_{\mu}^{\star} \nabla_{\mu}+m_{0}+i \gamma_{5} \tau_{3} \mu_{0},
$$

which has the self-adjointness property 22

$$
\tau_{1} \gamma_{5} D_{W D} \gamma_{5} \tau_{1}=D_{W D}^{\dagger}
$$

It follows then that the propagator for the upper and lower component of the quark doublet, $\psi_{l}(x)$, call them $S_{u}(x, y)$ and $S_{d}(x, y)$ respectively, satisfy the relations

$$
\gamma_{5} S_{u}(x, y) \gamma_{5}=S_{d}^{\dagger}(y, x), \quad \gamma_{5} S_{d}(x, y) \gamma_{5}=S_{u}^{\dagger}(y, x)
$$

This means that any baryon two-point correlator which is invariant under the interchange of the quark states in the quark doublet combined with hermitian conjugation, leading to the degeneracies mentioned above. An argument of this type has been given in Ref. [19].

The same can also be shown in a chiral Lagrangian treatment, as must be the case. Now one of the symmetries of tmLQCD with two flavor-degenerate quarks and $\tau_{3}$-twisting is the pseudo-parity transformation, $\mathcal{P}_{F}^{1}$, where ordinary parity is combined with a flavor exchange [5],

$$
\mathcal{P}_{F}^{1}:\left\{\begin{array}{l}
U_{0}(x) \rightarrow U_{0}\left(x_{P}\right), \quad x_{P}=(-\mathbf{x}, t) \\
U_{k}(x) \rightarrow U_{k}^{\dagger}\left(x_{P}\right), \quad k=1,2,3 \\
\psi_{l}(x) \rightarrow i \tau_{1} \gamma_{0} \psi_{l}\left(x_{P}\right) \\
\bar{\psi}_{l}(x) \rightarrow-i \bar{\psi}_{l}\left(x_{P}\right) \gamma_{0} \tau_{1}
\end{array}\right.
$$

where $U_{\mu}$ are the lattice gauge fields. At the level of $\mathrm{HB} \chi \mathrm{PT}$, this is manifested as the invariance of the chiral Lagrangian under the transformations

$$
p(x) \leftrightarrow n\left(x_{P}\right), \quad \Delta^{++,-}(x) \leftrightarrow \Delta^{-,++}\left(x_{P}\right), \quad \Delta^{+, 0}(x) \leftrightarrow \Delta^{0,+}\left(x_{P}\right), \quad \otimes_{k=1}^{F} \mathcal{O}_{k}(x) \rightarrow \otimes_{k=1}^{F} \tau_{1} \mathcal{O}_{k}\left(x_{p}\right) \tau_{1},
$$

where for an operator in the chiral Lagrangian, $\mathcal{O}_{k}$ is any operator matrix that contracts with the flavor indices of the the nucleon $(N)$ or delta $\left(T_{\mu}\right)$ fields in the operator. If the $N$ or the $T_{\mu}$ fields contained in an operator have a total 
of $2 \mathrm{~F}$ flavor indices, $\otimes_{k=1}^{F} \mathcal{O}_{k}$ is the tensor product of $F$ operator matrices which contract with the $F$ distinct pairs of these flavor indices. The degeneracies in the nucleons and the delta multiplets discussed above would then follow if all the operators in the chiral Lagrangian that contribute to the baryon masses have a structure that satisfies the condition

$$
\otimes_{k=1}^{F} \mathcal{O}_{k}(x)=\otimes_{k=1}^{F} \tau_{1} \mathcal{O}_{k}\left(x_{P}\right) \tau_{1}
$$

Consider first the case for the nucleons. Since the nucleon fields are vectors in flavor space, we can take $F=1$ without loss of generality (the nucleon fields can only couple to one operator matrix). Since the chiral Lagrangian is built with just $\mathcal{M}_{ \pm}, \mathcal{W}_{ \pm}^{t w}, \mathcal{A}_{\mu}$, and $\mathcal{V}_{\mu}$, the operator matrix $\mathcal{O}_{k}$ can only be constructed from combinations of these fields. We need not consider combinations involving just $\mathcal{M}_{+}$and $\mathcal{W}_{+}^{t w}$, since the flavor structure of both is trivial, i.e. proportional to the identity. We also need not consider mass contributions arising from pion loops, because they must have the same flavor structure as the tree level local counterterms used to cancel the divergences in these loops. Therefore, we do not have to consider operators involving $\mathcal{A}_{\mu}$ and $\mathcal{V}_{\mu}$, which give rise to mass contributions only through pion-nucleon interactions. This leaves us with only combinations involving $\mathcal{M}_{-}$and $\mathcal{W}_{-}^{t w}$ as possible candidates to break the degeneracy in the nucleons. As was discussed in Sec. IC because of the parity-flavor symmetry of tmLQCD, $\mathcal{O}_{k}$ can not contain just a single $\mathcal{M}_{-}$or $\mathcal{W}_{-}^{t w}$, but must always have an even number from the set $\left\{\mathcal{M}_{-}, \mathcal{W}_{-}^{t w}\right\}$. Now any such combination would indeed have a pure tree level part, however, it is also proportional to the identity in flavor space. Thus there is no operator matrix, $\mathcal{O}_{k}$, that one can construct which violates the condition $\mathcal{O}_{k}(x)=\tau_{1} \mathcal{O}_{k}\left(x_{P}\right) \tau_{1}$.

The arguments for the case of the deltas runs similar to that for the nucleons. For the same reason given in the nucleon case, we need not consider operator structures that involve $\mathcal{M}_{+}, \mathcal{W}_{+}^{t w}, \mathcal{A}_{\mu}$, and $\mathcal{V}_{\mu}$. We need only consider operator structures involving an even number from the set $\left\{\mathcal{M}_{-}, \mathcal{W}_{-}^{t w}\right\}$. For the deltas, $F$ can be three since each delta field has three flavor indices. But since two of the $\mathcal{O}_{k}$ in $\otimes_{k=1}^{3} \mathcal{O}_{k}$ must come from the set $\left\{\mathcal{M}_{-}, \mathcal{W}_{-}^{t w}\right\}$ to satisfy the parity-flavor symmetry of tmLQCD, we can take $F$ to be at most two without loss of generality. Now each of $\mathcal{M}_{-}$and $\mathcal{W}_{-}^{t w}$ has a tree level part that is proportional to $\tau_{3}$, thus, under $\mathcal{P}_{F}^{1}, \mathcal{O}_{1} \otimes \mathcal{O}_{2}$ where $\mathcal{O}_{k}$ can be either $\mathcal{M}_{-}$ or $\mathcal{W}_{-}^{t w}$, satisfies the symmetry condition (64). Therefore, one can not construct operators for the deltas that break the degeneracy between the pairs in the delta multiplet.

\section{NUCLEON AND DELTA MASSES AWAY FROM THE ISOSPIN LIMIT}

In this section, we present results for mass corrections due to twisting away from the isospin limit, where the quarks are now mass non-degenerate. To the order we work, the corrections due to the mass splitting come in only at tree level. For clarity, we will only point out the change arising from the quark mass splitting; we will not repeat the discussion on the nucleon and delta masses that are the same both in and away from the isospin limit.

\section{A. The flavor-diagonal basis for the mass matrix at $\mathcal{O}\left(\varepsilon^{4}\right)$}

The natural choice for splitting the quark doublet is to use the real and flavor-diagonal Pauli matrix, $\tau_{3}$, since the quark states one uses on the lattice correspond to the quarks in QCD in the continuum limit. But as was discussed in Sec. [A above, twisting can not be implemented with $\tau_{3}$ in this case (the fermionic determinant would be complex otherwise), and so $\tau_{1}$ is used instead. This means that the quark mass matrix in $\mathcal{L}_{\text {eff }}$ given in Eq. (5),$m+i \mu \gamma_{5} \tau_{1}-\epsilon_{q} \tau_{3}$, can never be made flavor-diagonal through an appropriate change of basis if both the twist and the mass splitting are non-vanishing, because $\tau_{1}$ and $\tau_{3}$ can not be simultaneously diagonalized.

Since the twist is implemented by a flavor nondiagonal Pauli matrix away from the isospin limit, flavor mixings are induced for non-zero twist: the quark states in tmLQCD are now linear combinations of the physical quarks of continuum QCD. At the level of the chiral effective theory, this manifests itself in that the hadronic states described by the tm $\chi \mathrm{PT}$ Lagrangian are linear combinations of the continuum QCD hadronic states we observe, viz. the pions, nucleons, deltas, etc.

If the effects from twisting are perturbative as compared to the isospin breaking effects, the hadronic states described by tm $\chi \mathrm{PT}$ will be "perturbatively close" to their corresponding continuum QCD states, i.e. the difference between them is small compared to the scales in the theory (see Appendix B for an explicit demonstration). In this case, we can still extract QCD observables directly from tm $\chi \mathrm{PT}$, as the corrections will be perturbative in the small expansion parameter. However, if the twisting effects are on the same order as the isospin breaking effects so that the flavor 
mixings are large, these corrections will not be perturbative. ${ }^{8}$ Nevertheless, one can still extract information for the QCD observables: One can still measure the masses of these tmLQCD hadronic states in lattice simulations, and one can fit these to the analytic expressions for these masses calculated in tm $\chi \mathrm{PT}$ to extract the values of the LECs. The LECs associated with the continuum $\chi \mathrm{PT}$ contributions have the same numerical values as in tm $\chi \mathrm{PT}$. Therefore, if one determines these from tmLQCD simulations, one knows the masses of the QCD hadronic states.

At the order we work, flavor mixings are manifested in the appearance of flavor non-conserving pion-baryon vertices in the Feynman rules of tmHB $\chi \mathrm{PT}$, and in that the baryon mass matrix is not flavor-diagonal. Since we work in the physical pion basis where the twist is carried by the Wilson spurion (now flavor non-diagonal) instead of the mass spurion (now flavor-diagonal), flavor mixings can only arise from operators with one or more insertions of the Wilson spurion field. Because of this, the flavor non-conserving pion-baryon vertices and the non-diagonal terms in the mass matrix must be proportional to $a$, the lattice spacing, and so must vanish in the continuum limit where the effects of the twist are fake and can be removed by a suitable chiral change of variables [1, 2] . ${ }^{9}$

For the nucleons, flavor mixings induce only unphysical flavor non-conserving pion-nucleon vertices which vanish in the continuum limit; the nucleon matrix is still flavor diagonal at the order we work. In fact, this is true to all orders in $\operatorname{tmHB} \chi \mathrm{PT}$. The reason is the same as that given in Sec. IIIC. We need only consider the tree level part of the possible operator structures that one can construct from the spurion fields in $\operatorname{tmHB} \chi \mathrm{PT}$. Now the only spurion field that has a tree level part with non-diagonal flavor structure is $\mathcal{W}_{-}^{t w}$, and as we discussed above, it must be paired either with another $\mathcal{W}_{-}^{t w}$ or with $\mathcal{M}_{-}$, which renders the flavor structure of the tree level part of the combination trivial. Thus we may take the basis of nucleons used in the tmHB $\chi \mathrm{PT}$ Lagrangian as the physical nucleon basis.

For the deltas, not only are there flavor non-conserving pion-delta vertices, at the order we work, the delta mass matrix is already flavor nondiagonal at tree level. This happens for the deltas because the tensor nature of the $T_{\mu}$ field allows more freedom in the way the flavor structure of the delta operator can be constructed. Thus, in order to have only physical tree level mass terms for the deltas, we must change to a basis where the delta mass matrix is diagonal, which can now only be done order by order.

When diagonalizing the delta mass matrix, we need, in principle, to diagonalize the mass matrix that contains all the mass contributions from both tree and loop level to the order that one works. But we find the difference between diagonalizing the delta mass matrix including both tree and loop level contributions at the order we work, and diagonalizing that with only the tree level mass contributions, give rise to corrections only to the loop level mass contributions, which are higher order than we work. Thus, we will diagonalize the delta mass matrix containing just the tree level mass terms in our calculation for the delta masses.

To the order we work, if the tree level mass is given by

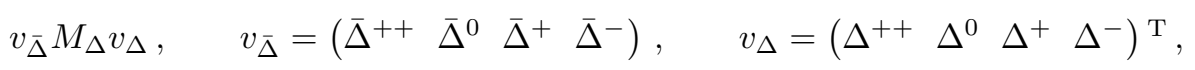

where $v_{\bar{\Delta}}$ and $v_{\Delta}$ are vectors of the delta basis states used in the tmHB $\chi \mathrm{PT}$ Lagrangian, and $M_{\Delta}$ is the tree level mass matrix, the physical delta basis is given by

$$
v_{\Delta}^{\prime}=S^{-1} \cdot v_{\Delta}, \quad v_{\bar{\Delta}}^{\prime}=v_{\bar{\Delta}} \cdot S,
$$

where $S$ is the matrix of eigenvectors of $M_{\Delta}$ such that

$$
S \cdot M_{\Delta} \cdot S^{-1}=\mathcal{D}
$$

with $\mathcal{D}$ the corresponding diagonal eigenvalue matrix. This implies that

$$
v_{\bar{\Delta}} M_{\Delta} v_{\Delta}=\left(v_{\bar{\Delta}}^{\prime} \cdot S^{-1}\right) \cdot\left(S \cdot \mathcal{D} \cdot S^{-1}\right) \cdot\left(S \cdot v_{\Delta}^{\prime}\right)=v_{\bar{\Delta}}^{\prime} \mathcal{D} v_{\Delta}^{\prime} .
$$

The full details of the diagonalization are provided in Appendix $B$ In the following sections, we will work in this basis for calculating the delta masses.

\footnotetext{
${ }^{8}$ A qualitative guide to the size of the flavor mixings can be found in the ratios of two-point correlation functions. Define the ratio of QCD delta states by$$
R_{i j} \equiv \frac{\left\langle\Delta^{i} \Delta^{j}\right\rangle+\left\langle\Delta^{j} \Delta^{i}\right\rangle}{\left\langle\Delta^{i} \Delta^{i}\right\rangle+\left\langle\Delta^{j} \Delta^{j}\right\rangle}
$$

Flavor mixing should be small if the off diagonal elements of $R_{i j}$ are small. To determine the size of the flavor mixings quantitatively, one has to look at the splitting in the delta multiplet. We will discuss further in the text below.

9 This shows again the convenience of the pion physical basis, where all the effects of symmetry breaking in the lattice theory are parametrized and contained in the Wilson spurion fields, which then vanish as the symmetries are restored in the continuum limit.
} 
The Feynman rules in the new basis are obtained from the same tmHB $\chi \mathrm{PT}$ Lagrangian given above in Sec. [IC but with each of the delta flavor states now rewritten in terms of the new delta flavor states given by the defining relations (66). Note that changing to the new delta basis induces new unphysical flavor non-conserving vertices in the delta interaction terms given in (26), because in terms of the new basis states, flavors are mixed. However, these flavor mixing components are proportional to the off-diagonal elements of $S$, which are proportional to the lattice spacing as well as the twist angle (see Appendix B). Thus they vanish in the limit of vanishing twist or lattice spacing, and so the unphysical vertices arising from them also vanish in these limits.

We note and reiterate here that in the isospin limit, this order by order mass matrix diagonalization is unnecessary as one can always rotate to a basis where the twist is flavor-diagonal from the outset, and issues of flavor nonconserving vertices and non-diagonal mass matrices due to flavor mixings do not arise. ${ }^{10}$

\section{B. The nucleon masses}

Away from the isospin limit, the first change caused by the mass splitting occurs in the continuum mass contribution $M_{N_{i}}^{(1)}$, since the quark masses

$$
m_{u}=m_{q}-\epsilon_{q}, \quad m_{d}=m_{q}+\epsilon_{q}, \quad m_{q}, \epsilon_{q}>0,
$$

are no longer equal.

At the order we work, the only other change due to the mass splitting appears at $\mathcal{O}\left(\varepsilon^{4}\right)$ in the contribution from the $\mathcal{O}(a \mathrm{~m})$ nucleon operator with coefficient $n^{W M_{+}}$given in Eq. (34). In the isospin limit, its contribution to $\delta M_{N_{i}}^{(2)}(\omega)$ is proportional to $m_{q}$, but away from the isospin limit, it becomes

$$
2 n_{1}^{W M_{+}} a \Lambda_{\chi} m_{q} \cos (\omega) \longrightarrow 2 n_{1}^{W M_{+}} a \Lambda_{\chi} m_{i} \cos (\omega),
$$

where

$$
m_{i}=\left\{\begin{array}{ll}
m_{u} & \text { for } i=p \\
m_{d} & \text { for } i=n
\end{array} .\right.
$$

The corrections to the nucleon masses from the effects of lattice discretization and twisting are otherwise the same as those given in Eqs. (43) and (46).

Note that the nucleon masses are automatically $\mathcal{O}(a)$ improved, just as in the isospin limit.

\section{The delta masses}

Away from the isospin limit $\left(\epsilon_{q} \neq 0\right)$, we calculate the delta mass and mass corrections in the basis where the delta mass matrix is diagonal to the order we work. This diagonalization is worked out in Appendix B where we have obtained general expressions for the new delta basis that are valid in the range from $\epsilon_{q}=0$ to $\epsilon_{q} \sim a \Lambda_{\mathrm{QCD}}^{2}$. Here, we present the case where $\epsilon_{q}>0$ and $\epsilon_{q} \sim m_{q} \sim a \Lambda_{\mathrm{QCD}}^{2} \gg a^{2} \Lambda_{\mathrm{QCD}}^{3}$, which is a regime that simulations in the near future can probe. To the order we work, we may take the new delta basis states in this regime to be

$$
\begin{array}{rlrl}
T_{1}^{\prime} \leftrightarrow\left|\Delta_{1}\right\rangle & =\mathcal{C}_{1}\left[\left|\Delta^{++}\right\rangle+\frac{\sqrt{3} B}{4 A}\left|\Delta^{0}\right\rangle\right], & & T_{3}^{\prime} \leftrightarrow\left|\Delta_{3}\right\rangle=\mathcal{C}_{3}\left[\left(1+\frac{B}{4 A}\right)\left|\Delta^{0}\right\rangle-\frac{\sqrt{3} B}{4 A}\left|\Delta^{++}\right\rangle\right], \\
T_{2}^{\prime} \leftrightarrow\left|\Delta_{2}\right\rangle=\mathcal{C}_{2}\left[\left|\Delta^{+}\right\rangle+\frac{\sqrt{3} B}{4 A}\left|\Delta^{-}\right\rangle\right], & T_{4}^{\prime} \leftrightarrow\left|\Delta_{4}\right\rangle=\mathcal{C}_{4}\left[\left(1-\frac{B}{4 A}\right)\left|\Delta^{-}\right\rangle-\frac{\sqrt{3} B}{4 A}\left|\Delta^{+}\right\rangle\right],
\end{array}
$$

where $T_{i}^{\prime}=\Delta_{i}$ denote the deltas in the new basis, $\mathcal{C}_{i}$ are normalization factors, and

$$
A=2 \epsilon_{q}\left(\gamma_{M}+t_{1}^{W M_{+}} \frac{a \Lambda_{Q C D}^{2}}{\Lambda_{\chi}} \cos (\omega)\right), \quad B=t_{2}^{W_{-}} a^{2} \Lambda_{Q C D}^{3} \sin ^{2}(\omega) .
$$

\footnotetext{
${ }^{10}$ In fact, as is shown in Appendix [B] if one insists on remaining in the basis where the twist in flavor non-diagonal, one would find that
} the unphysical terms arising from flavor mixings do not vanish in the continuum limit. 
Note that $A \sim \mathcal{O}\left(\varepsilon^{2}\right)$ and $B \sim \mathcal{O}\left(\varepsilon^{4}\right)$ in our power counting, so $B / A \sim \mathcal{O}\left(\varepsilon^{2}\right)$ and the effects of the flavor mixings is perturbative.

The masses of these states are comprised of the continuum expressions given in Ref. [29] and corrections due to the effects of discretization and twisting. Note the continuum expressions for the delta masses here are necessarily changed from that in the isospin limit because $m_{u} \neq m_{d}$. The mass corrections due to the effects of lattice discretization and twisting, come in at tree level; the loop contributions remain unchanged from that in the isospin limit. The tree level mass contributions to the order we work have been worked out in Eq. (B7), in the process of diagonalization. We list here the full delta mass corrections to $\mathcal{O}\left(\varepsilon^{4}\right)$, which we denote by $\delta M_{i}$, to the mass of the delta state denoted by $T_{i}^{\prime}$ :

$$
\begin{aligned}
\delta M_{i}(\omega)= & -4 \bar{\sigma}_{W} a \Lambda_{Q C D}^{2} \cos (\omega) \\
& +12 \bar{\sigma}_{W} \frac{a \Lambda_{\mathrm{QCD}}^{2}}{\Lambda_{\chi}^{2}} m_{\pi}^{2} \log \left(\frac{m_{\pi}^{2}}{\Lambda_{R}^{2}}\right) \cos (\omega)+4 g_{\Delta N}^{2}\left(\bar{\sigma}_{W}-\sigma_{W}\right) \frac{a \Lambda_{\mathrm{QCD}}^{2}}{\Lambda_{\chi}^{2}} \mathcal{J}\left(m_{\pi},-\Delta, \Lambda_{R}\right) \cos (\omega) \\
& +2 t_{1}^{W M_{+}} \frac{a \Lambda_{\mathrm{QCD}}^{2}}{\Lambda_{\chi}} \frac{m_{i}^{\prime}}{3} \cos (\omega)+4 t_{2}^{W M_{+}} \frac{a \Lambda_{\mathrm{QCD}}^{2}}{\Lambda_{\chi}} m_{q} \cos (\omega) \\
& +a^{2} \Lambda_{Q C D}^{3}\left(t+t_{v}\right)+a^{2} \Lambda_{Q C D}^{3}\left(4 t_{1}^{W_{+}} \cos ^{2}(\omega)-2 t_{1}^{W-} \sin ^{2}(\omega)+t_{2}^{W-} \delta_{i}^{\prime} \sin ^{2}(\omega)\right), \quad i=1, \ldots, 4,
\end{aligned}
$$

where

$$
m_{i}^{\prime}=\left\{\begin{array}{ll}
3 m_{u} & \text { for } i=1 \\
2 m_{u}+m_{d} & \text { for } i=2 \\
m_{u}+2 m_{d} & \text { for } i=3 \\
3 m_{d} & \text { for } i=4
\end{array}, \quad \delta_{i}^{\prime}=\left\{\begin{array}{rr}
0 & \text { for } i=1,4 \\
-\frac{2}{3} & \text { for } i=2,3
\end{array}\right.\right.
$$

Note that $\delta M_{i}(\omega)$ as given in Eq. (74), is the same as the sum of $\delta M_{T_{i}}^{(1)}$ and $\delta M_{T_{i}}^{(2)}$ as given in Eqs. (52) and (54) respectively, but with the changes

$$
2 t_{1}^{W M_{+}} \frac{a \Lambda_{\mathrm{QCD}}^{2}}{\Lambda_{\chi}} m_{q} \cos (\omega) \longrightarrow 2 t_{1}^{W M_{+}} \frac{a \Lambda_{\mathrm{QCD}}^{2}}{\Lambda_{\chi}} \frac{m_{i}^{\prime}}{3} \cos (\omega), \quad \delta_{T_{i}} \longrightarrow \delta_{i}^{\prime} .
$$

The full expressions for the delta masses can be obtained when the continuum contributions are included. To the order we work, one can obtain the complete mass expression for delta denoted by $T_{i}^{\prime}$ to $\mathcal{O}\left(\varepsilon^{4}\right)$ in tmHB $\chi \mathrm{PT}$ by adding the mass corrections, $\delta M_{i}(\omega)$, to the continuum mass of the delta denoted by $T_{i}$, whose expression can be found in Ref. [29].

We stress here that one can not take the isospin limit from any of the expressions give above in this subsection. They have been derived for $\epsilon_{q} \neq 0$ and with the assumption that the twisting effects are much smaller than the isospin breaking effects. One must use the general formulae given in Eqs. (B6) and (B7) when considering cases where these conditions are not true.

Observe that away from the isospin limit, the delta masses are also automatically $\mathcal{O}(a)$ improved at maximal twist ( $\omega=\pi / 2)$, as all terms proportional to $a$ in $\delta M_{i}$ are proportional to $\cos (\omega)$ as well. Hence, to the order we work, the contributions due to the isospin breaking are the same as that in the continuum at maximal twist.

\section{SUMMARY}

In this paper we have studied the mass spectrum of the nucleons and the deltas in tmLQCD with mass nondegenerate quarks using effective field theory methods. We have extended heavy baryon chiral perturbation theory for $S U(2)$ to include the effects of the twisted mass, and we have done so to $\mathcal{O}\left(\varepsilon^{4}\right)$ in our power counting, which includes operators of $\mathcal{O}\left(a \mathrm{~m}^{2}, a p^{2}, a^{2}\right)$. Using the resulting tmHB $\chi \mathrm{PT}$, we have calculated the nucleon and the delta masses to $\mathcal{O}\left(\varepsilon^{4}\right)$, and we found them to be automatically $\mathcal{O}(a)$ improved as expected from the properties of tmLQCD.

Because of the twisting, the vacuum is no longer aligned with the identity in flavor space, which has non-trivial effects on the physical excitations (pions) of the theory. Also, depending on whether the quarks are mass degenerate or not, the way twisting is implemented determines what the physical baryon states are in the theory. We have highlighted these subtleties when doing calculations in tmHB $\chi \mathrm{PT}$.

In order for the pions in the theory to be physical, we have to make a particular (non-anomalous) chiral change of variables to undo the twisting effects. This requires the knowledge of the twisting angle, but once that is determined, the physical pion basis can be determined $\grave{a}$ priori. However, whether or not the nucleons and deltas are physical must 
still be determined from the theory. In the isospin limit, both the nucleon and the delta mass matrices are diagonal, and so the nucleon and delta states contained in the $N$ and $T_{\mu}$ fields are physical. However, away from the isospin limit, only the nucleon mass matrix remains diagonal. Thus, the $N$ field can still be regarded as physical, but the physical deltas are now linear combinations of the flavor states contained in the $T_{\mu}$ field. This can be understood from the fact the at the quark level, the physical QCD states, the $u$ and $d$ quarks, are eigenstates of $\tau_{3}$ but not of $\tau_{1}$. So only in the isospin limit, where the twist can always be implemented by the flavor-diagonal Pauli matrix, $\tau_{3}$, are the states contained in the quark doublet physical quarks. Away from the isospin limit, the twist can not be implemented by $\tau_{3}$ anymore, and the eigenstates of the Hamiltonian of the theory are composed of linear combinations of the $u$ and $d$ quarks.

The physical states in $\operatorname{tm} \chi \mathrm{PT}$ are in general a mixture of those in the untwisted $\chi \mathrm{PT}$. The size of the mixture is determined by the relative sizes of the discretization effects, which are $\mathcal{O}\left(a^{2}\right)$, and the isospin splitting effects, which are $\mathcal{O}\left(\epsilon_{q}\right)$. In this work, we have given general expressions for the nucleon and delta masses with respect to this mixing of states that are valid in the range from $\epsilon_{q}=0$ to $\epsilon_{q} \sim a \Lambda_{\mathrm{QCD}}$.

The quantities which provided the motivation for this work and turned out to be most interesting are the mass splittings between the nucleons and between the deltas. We found that in the isospin limit, the nucleon masses do not split to any order in $\operatorname{tm} \chi \mathrm{PT}$, while the delta multiplet splits into two degenerate pairs. This can be understood from the symmetries of tmLQCD at the quark level, and as we have shown, also at the level of tm $\chi$ PT. The mass splitting between the multiplets, $M_{\Delta^{+, 0}}-M_{\Delta^{++},-}$, first arises from a tree level contribution at $\mathcal{O}\left(a^{2}\right)$, and it gives an indication of the size of the flavor breaking in tmLQCD. This splitting in the delta multiplet will be easier to calculate in lattice simulations than the corresponding quantity $m_{\pi_{3}}^{2}-m_{\pi_{1,2}}^{2}$ in the meson sector [18], since it involves no quark disconnected diagrams.

Twisted mass $\mathrm{HB} \chi \mathrm{PT}$ can also be extended to partially quenched theories (such extension of $\operatorname{tm} \chi \mathrm{PT}$ for pions has recenly been done [43]). This will be useful in the near future as numerical studies of tmLQCD move from quenched simulations to the more realistic, if more computationally demanding, partially quenched simulations.

\section{Acknowledgments}

We thank Stephen Sharpe for many useful discussions, and Brian Tiburzi for helpful comments. This research was supported by the U.S. Department of Energy Grant Nos. DE-FG02-96ER40956 (J.M.S.W.), and DE-FG03-97ER41014 (A.W.-L.).

\section{APPENDIX A: ABSENCE OF ADDITIONAL DIMENSION FIVE SYMMETRY BREAKING OPERATORS INDUCED BY THE MASS SPLITTING}

In this appendix, we show that the mass splitting does not induce any symmetry breaking terms in the effective continuum Lagrangian at the quark level at quadratic order. The (mass) dimension six operators in the Symanzik Lagrangian we can drop for the same reason given in Ref. [10], since they are either of too high order (cubic or higher in our expansion) or they do not break the symmetries further than those of lower dimensions. For dimension five operators, we will show that the only allowable terms by the symmetries of the lattice theory are those that either vanish by the equations of motion, or can be removed by suitable $\mathcal{O}(a)$ redefinitions of the parameters in $\mathcal{L}_{0}$, the effective Lagrangian in the continuum limit (the lowest order effective Lagrangian).

In the mass-degenerate case [10], the only dimension five operator that appears is the Pauli term. Since in the limit of vanishing mass splitting (the isospin limit) the mass non-degenerate theory must be the same as the massdegenerate theory, any additional operators induced by the mass splitting must be proportional to the mass splitting. These can only be of the form

$$
\begin{array}{llll}
\epsilon_{q}^{2} \bar{\psi} \mathcal{O}_{0} \psi: & \mathcal{O}_{0}=\Gamma_{0} \backslash\{\mathbb{1}\}, \quad \Gamma_{0}=\left\{\mathbb{1}, \tau_{k}, \gamma_{5}, \gamma_{5} \tau_{k}\right\}, & k=1,2,3, & \operatorname{dim}\left[\mathcal{O}_{0}\right]=0, \\
\epsilon_{q} \bar{\psi} \mathcal{O}_{1} \psi: & \mathcal{O}_{1}=\left\{\not D \Gamma_{0}, m \Gamma_{0}, \mu \Gamma_{0}\right\} \backslash\left\{\not D \tau_{3}, m \tau_{3}\right\}, & & \operatorname{dim}\left[\mathcal{O}_{1}\right]=1,
\end{array}
$$

where the notation " $P \backslash Q$ " means "the set $\mathrm{P}$ excluding the set Q". The quantities $\mathcal{O}_{0}$ and $\mathcal{O}_{1}$ are all the possible independent structures with the correct dimension, which do not lead to dimension five operators vanishing by the equations of motion, or are not removable by redefinitions of parameters in $\mathcal{L}_{0}$. However, none of these operators are allowed under the symmetries of the lattice theory. Specifically, they are forbidden by charge conjugation $(\mathcal{C})$ and the pseudo-parity transformations that combine the ordinary parity transformation $(\mathcal{P})$ with a parameter sign change

$$
\widetilde{\mathcal{P}} \equiv \mathcal{P} \times(\mu \rightarrow-\mu)
$$




\begin{tabular}{|c|c|c|c|c|}
\hline \hline Structure & $\mathcal{C}$ & $\mathcal{P}_{F}^{3}$ & $\widetilde{\mathcal{P}}$ & $\mathcal{P}_{F, \epsilon_{q}}^{2}$ \\
\hline $\mathcal{O}_{0}$ & $\tau_{2}, \gamma_{5} \tau_{2}$ & $\tau_{1}, \gamma_{5}, \gamma_{5} \tau_{3}$ & $\gamma_{5} \tau_{1}$ & $\tau_{3}$ \\
\hline $\mathcal{O}_{1}$ & $\not D \gamma_{5} \times\left\{\mathbb{1}, \tau_{1}, \tau_{3}\right\}$ & $\not D \tau_{1}, \not D \tau_{2}$ & $\not D \gamma_{5} \tau_{2}$ & \\
& $m \tau_{2}, m \gamma_{5} \tau_{2}$ & $m \times\left\{\tau_{1}, \gamma_{5}, \gamma_{5} \tau_{3}\right\}$ & $m \gamma_{5} \tau_{1}$ & \\
& $i \mu \tau_{2}, i \mu \gamma_{5} \tau_{2}$ & $i \mu \times\left\{\tau_{1}, \gamma_{5}, \gamma_{5} \tau_{3}\right\}$ & $i \mu \tau_{3}$ & $i \mu \gamma_{5} \tau_{1}$ \\
\hline \hline
\end{tabular}

TABLE I: The structures of the dimension five operators that are non-vanishing by the equations of motion and non-removable by parameter redefinitions. They are classified by the symmetries that forbid them.

or a flavor exchange or both

$$
\mathcal{P}_{F, \epsilon_{q}}^{2} \equiv \mathcal{P}_{F}^{2} \times\left(\epsilon_{q} \rightarrow-\epsilon_{q}\right), \quad \mathcal{P}_{F}^{3},
$$

where

$$
\mathcal{P}_{F}^{2,3}:\left\{\begin{array}{l}
U_{0}(x) \rightarrow U_{0}\left(x_{P}\right), \quad x_{P}=(-\mathbf{x}, t) \\
U_{k}(x) \rightarrow U_{k}^{\dagger}\left(x_{P}\right), \quad k=1,2,3 \\
\psi(x) \rightarrow i \tau_{2,3} \gamma_{0} \psi\left(x_{P}\right) \\
\bar{\psi}(x) \rightarrow-i \bar{\psi}\left(x_{P}\right) \gamma_{0} \tau_{2,3}
\end{array},\right.
$$

and $U_{\mu}$ are the lattice link fields. Note that we have displayed the symmetries of the lattice theory [5, 22] in the form which applies to the effective continuum theory.

In Table 1, we show explicitly which symmetry forbids each of the possible structures of $\mathcal{O}_{0}$ and $\mathcal{O}_{1}$ listed in a1). ${ }^{11}$ We group the operators in columns according to the symmetry under which they are forbidden.

The conclusion of the above discussion is that the mass splitting does not induce any additional operators that do not vanish by the equations of motion, or can not be removed by redefinitions of the parameters in the theory. Thus beyond $\mathcal{L}_{0}$, the effective continuum Lagrangian contains only the Pauli term to the order we work, exactly as in the mass-degenerate case.

\section{APPENDIX B: DIAGONALIZATION OF THE DELTA MASS MATRIX}

Here we diagonalize the tree level mass matrix for the delta states. We reiterate that the difference between first diagonalizing the tree level mass contributions, then calculating loop effects, versus calculating the loop contributions then diagonalizing, is of higher order than we work. To proceed, first we list all the independent operators to $\mathcal{O}\left(\varepsilon^{4}\right)$ that have tree level mass contributions,

$$
\begin{array}{rll}
\mathcal{O}\left(\varepsilon^{2}\right): & \left(\bar{T}_{\mu} \mathcal{M}_{+} T_{\mu}\right), \quad\left(\bar{T}_{\mu} T_{\mu}\right) \operatorname{tr}\left(\mathcal{M}_{+}\right), \quad\left(\bar{T}_{\mu} T_{\mu}\right) \operatorname{tr}\left(\mathcal{W}_{+}^{t w}\right) \\
\mathcal{O}(a \mathrm{~m}): & \left(\bar{T}_{\mu} \mathcal{M}_{+} T_{\mu}\right) \operatorname{tr}\left(\mathcal{W}_{+}^{t w}\right), \quad\left(\bar{T}_{\mu} T_{\mu}\right) \operatorname{tr}\left(\mathcal{W}_{+}^{t w}\right) \operatorname{tr}\left(\mathcal{M}_{+}\right) \\
\mathcal{O}\left(a^{2}\right): & \left(\bar{T}_{\mu} T_{\mu}\right) \operatorname{tr}\left(\mathcal{W}_{+}^{t w}\right) \operatorname{tr}\left(\mathcal{W}_{+}^{t w}\right), \quad\left(\bar{T}_{\mu} T_{\mu}\right) \operatorname{tr}\left(\mathcal{W}_{-}^{t w} \mathcal{W}_{-}^{t w}\right), \quad \bar{T}_{\mu}^{k j i}\left(\mathcal{W}_{-}^{t w}\right)^{i i^{\prime}}\left(\mathcal{W}_{-}^{t w}\right)^{j j^{\prime}} T_{\mu}^{i^{\prime} j^{\prime} k}
\end{array}
$$

The tree level delta mass matrix at the order we work, $M_{\Delta}$, is then given by

$$
\begin{aligned}
v_{\bar{\Delta}} M_{\Delta} v_{\Delta} & =v_{\bar{\Delta}}\left(\begin{array}{cccc}
-A+C & -\frac{B}{\sqrt{3}} & 0 & 0 \\
-\frac{B}{\sqrt{3}} & \frac{1}{3}(A-2 B)+C & 0 & 0 \\
0 & 0 & -\frac{1}{3}(A+2 B)+C & -\frac{B}{\sqrt{3}} \\
0 & 0 & -\frac{B}{\sqrt{3}} & A+C
\end{array}\right) v_{\Delta}, \\
& =v_{\bar{\Delta}}\left\{C \mathbb{1}_{4 \times 4}+K_{\Delta}\right\} v_{\Delta},
\end{aligned}
$$

\footnotetext{
11 Most of what we show can be readily inferred from [22]. What is new here is the need for $\widetilde{\mathcal{P}}$, and the use of $\mathcal{P}_{F, \epsilon_{q}}^{2}$.
} 
where the vectors $v_{\bar{\Delta}}$ and $v_{\Delta}$ are vectors of the (QCD) delta basis states,

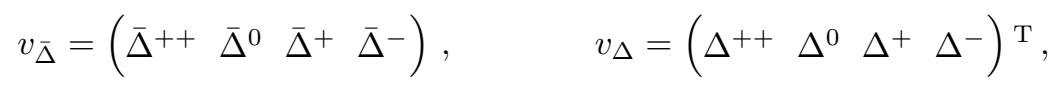

and

$$
K_{\Delta}=\left(\begin{array}{cccc}
-A & -\frac{B}{\sqrt{3}} & 0 & 0 \\
-\frac{B}{\sqrt{3}} & \frac{1}{3}(A-2 B) & 0 & 0 \\
0 & 0 & -\frac{1}{3}(A+2 B) & -\frac{B}{\sqrt{3}} \\
0 & 0 & -\frac{B}{\sqrt{3}} & A
\end{array}\right)
$$

The entries in $M_{\Delta}$ are given by

$$
\begin{aligned}
A= & 2 \epsilon_{q}\left(\gamma_{M}+t_{1}^{W M_{+}} \frac{a \Lambda_{Q C D}^{2}}{\Lambda_{\chi}} \cos (\omega)\right), \quad B=t_{2}^{W-} a^{2} \Lambda_{Q C D}^{3} \sin ^{2}(\omega), \\
C= & 2 m_{q}\left(\gamma_{M}-2 \bar{\sigma}_{M}\right)-4 \bar{\sigma}_{W} a \Lambda_{Q C D}^{2} \cos (\omega)+2 m_{q}\left(t_{1}^{W M_{+}}+2 t_{2}^{W M_{+}}\right) \frac{a \Lambda_{Q C D}^{2}}{\Lambda_{\chi}} \cos (\omega) \\
& +a^{2} \Lambda_{Q C D}^{3}\left(t+t_{v}\right)+a^{2} \Lambda_{Q C D}^{3}\left(4 t_{1}^{W_{+}} \cos ^{2}(\omega)-2 t_{1}^{W-} \sin ^{2}(\omega)\right) .
\end{aligned}
$$

Note that to the accuracy we work, $\omega$ can be either $\omega_{0}$ or the non-perturbatively determined twist angle.

Except for the operators

$$
\left(\bar{T}_{\mu} \mathcal{M}_{+} T_{\mu}\right), \quad\left(\bar{T}_{\mu} \mathcal{M}_{+} T_{\mu}\right) \operatorname{tr}\left(\mathcal{W}_{+}^{t w}\right), \quad \bar{T}_{\mu}^{k j i}\left(\mathcal{W}_{-}^{t w}\right)^{i i^{\prime}}\left(\mathcal{W}_{-}^{t w}\right)^{j j^{\prime}} T_{\mu}^{i^{\prime} j^{\prime} k},
$$

which contribute to $K_{\Delta}$, all other operators listed in (B1) above have trivial flavor structure, and so contribute to the identity part of $M_{\Delta}$. Hence, to diagonalize $M_{\Delta}$, we need only diagonalize $K_{\Delta}$. The orthogonal matrix that accomplishes this is

$$
S=\left(\begin{array}{cccc}
\frac{\left(2 A-B+2 X_{-}\right)^{1 / 2}}{2 X_{-}^{1 / 2}} & -\frac{\left(-2 A+B+2 X_{-}\right)^{1 / 2}}{2 X_{-}^{1 / 2}} & 0 & 0 \\
\frac{\sqrt{3} B}{2 X_{-}^{1 / 2}\left(2 A-B+2 X_{-}\right)^{1 / 2}} & \frac{\sqrt{3} B}{2 X_{-}^{1 / 2}\left(-2 A+B+2 X_{-}\right)^{1 / 2}} & 0 & 0 \\
0 & 0 & \frac{\left(2 A+B+2 X_{+}\right)^{1 / 2}}{2 X_{+}^{1 / 2}} & -\frac{\left(-2 A-B+2 X_{+}\right)^{1 / 2}}{2 X_{+}^{1 / 2}} \\
0 & 0 & \frac{\sqrt{3} B}{2 X_{+}^{1 / 2}\left(2 A+B+2 X_{+}\right)^{-1 / 2}} & \frac{\sqrt{3} B}{2 X_{+}^{1 / 2}\left(-2 A-B+2 X_{+}\right)^{-1 / 2}}
\end{array}\right)
$$

where $X_{ \pm}=\sqrt{A^{2} \pm A B+B^{2}}$, and each column of $S$ is a normalized eigenvector of $M_{\Delta}$ (and hence $K_{\Delta}$ also). The diagonal matrix one obtains after diagonalizing $M_{\Delta}$ is then

$$
\mathcal{D}=S^{-1} \cdot M_{\Delta} \cdot S=\frac{1}{3} \operatorname{diag}\left(\begin{array}{r}
-A-B-2 X_{-}+3 C \\
-A-B+2 X_{-}+3 C \\
A-B-2 X_{+}+3 C \\
A-B+2 X_{+}+3 C
\end{array}\right)
$$

where each entry in $\mathcal{D}$ is an eigenvalue of $M_{\Delta}$.

Now if $\epsilon_{q} \neq 0, A \neq 0$. Hence, since in our power counting $A \sim \mathcal{O}\left(\varepsilon^{2}\right)$ and $B \sim \mathcal{O}\left(\varepsilon^{4}\right)$, we may expand $X_{ \pm}$in the ratio of $B / A \sim \mathcal{O}\left(\varepsilon^{2}\right) \ll 1$ as

$$
X_{ \pm}=A \sqrt{1 \pm \frac{B}{A}+\frac{B^{2}}{A^{2}}}=A\left(1 \pm \frac{1}{2} \frac{B}{A}+\frac{3}{8} \frac{B^{2}}{A^{2}}+\mathcal{O}\left(\varepsilon^{6}\right)\right)
$$

from which it follows that

$$
S=\left(\begin{array}{cccc}
1 & -\frac{\sqrt{3} B}{4 A} & 0 & 0 \\
\frac{\sqrt{3} B}{4 A} & 1+\frac{B}{4 A} & 0 & 0 \\
0 & 0 & 1 & -\frac{\sqrt{3} B}{4 A} \\
0 & 0 & \frac{\sqrt{3} B}{4 A} & 1-\frac{B}{4 A}
\end{array}\right), \quad \mathcal{D}=\operatorname{diag}\left(\begin{array}{c}
-A+C \\
\frac{A}{3}-\frac{2 B}{3}+C \\
-\frac{A}{3}-\frac{2 B}{3}+C \\
A+C
\end{array}\right)
$$


up to corrections of $\mathcal{O}\left(\varepsilon^{4}\right)$ for $S$ and $\mathcal{O}\left(\varepsilon^{6}\right)$ for $\mathcal{D}$.

If $\epsilon_{q}=0$, i.e. in the isospin limit, $A=0$ and $X_{ \pm}=B$. In this case, one can not find $S$ and $\mathcal{D}$ in the isospin limit by taking the limit $A \rightarrow 0$ in (B9), since expansion in the ratio of $B / A$ is clearly not valid. Instead, one has to go back to Eq. (B6) and Eq. (B7), which in the isospin limit reduce to

$$
S=\left(\begin{array}{cccc}
\frac{1}{2} & -\frac{\sqrt{3}}{2} & 0 & 0 \\
\frac{\sqrt{3}}{2} & \frac{1}{2} & 0 & 0 \\
0 & 0 & \frac{\sqrt{3}}{2} & -\frac{1}{2} \\
0 & 0 & \frac{1}{2} & \frac{\sqrt{3}}{2}
\end{array}\right), \quad \mathcal{D}=\operatorname{diag}\left(\begin{array}{r}
-B+C \\
\frac{B}{3}+C \\
-B+C \\
\frac{B}{3}+C
\end{array}\right)
$$

and the eigenvalues contained in $\mathcal{D}$ given in Eq. (B10) are the masses of the deltas at tree level in the isospin limit given in Eq. (52) and Eq. (54). Note that as discussed in the text, in the isospin limit, we need not perform any mass matrix diagonalization at all, since we can simply rotate from the outset to the basis where the twist is implemented by the diagonal $\tau_{3}$.

The new delta basis states are defined by

$$
v_{\Delta}^{\prime}=S^{-1} \cdot v_{\Delta}, \quad v_{\bar{\Delta}}^{\prime}=v_{\bar{\Delta}} \cdot S
$$

in which the delta mass matrix is diagonal to the order we work. By writing the old (unprimed) delta basis states in terms of the new (primed) basis states using the defining relations given above, i.e.

$$
v_{\Delta}=S \cdot v_{\Delta}^{\prime}, \quad v_{\bar{\Delta}}=v_{\bar{\Delta}}^{\prime} \cdot S^{-1},
$$

the Lagrangian in the new delta basis can be obtained. Note that in the case where $\epsilon_{q} \neq 0$, the new basis states contained in $v_{\Delta}^{\prime}$ are "perturbatively close" to those contained in the $v_{\Delta}$, i.e. the difference is $\mathcal{O}\left(\varepsilon^{2}\right)$ as can be easily seen from Eq. (B99). This is of course not true if we are in a region where $B \sim A$, or $\epsilon_{q} \sim a^{2} \Lambda_{\mathrm{QCD}}^{3}$.

[1] R. Frezzotti, P. A. Grassi, S. Sint and P. Weisz, Nucl. Phys. Proc. Suppl. 83, 941 (2000).

[2] R. Frezzotti, P. A. Grassi, S. Sint and P. Weisz [Alpha collaboration], JHEP 0108, 058 (2001).

[3] R. Frezzotti, "Twisted mass lattice QCD", plenary talk at Lattice 2004, hep-lat/0409138

[4] A. D. Kennedy, "Algorithms for lattice QCD with dynamical fermions," plenary talk at Lattice 2004, hep-lat/0409167

[5] R. Frezzotti and G. C. Rossi, JHEP 0408, 007 (2004).

[6] C. Pena, S. Sint and A. Vladikas, JHEP 0409, 069 (2004).

[7] R. Frezzotti and G. C. Rossi, JHEP 0410, 070 (2004).

[8] G. Münster and C. Schmidt, Europhys. Lett. 66, 652 (2004).

[9] L. Scorzato, Eur. Phys. J. C 37, 445 (2004).

[10] S. R. Sharpe and J. M. S. Wu, Phys. Rev. D 70, 094029 (2004).

[11] S. Sharpe and R. Singleton, Jr, Phys. Rev. D 58, 074501 (1998).

[12] G. Rupak and N. Shoresh, Phys. Rev. D 66, 054503 (2002).

[13] O. Bär, G. Rupak and N. Shoresh, Phys. Rev. D 70, 034508 (2004).

[14] G. Münster, JHEP 0409, 035 (2004).

[15] G. Münster, C. Schmidt and E. E. Scholz, hep-lat/0409066

[16] S. Sharpe and J. Wu, Nucl. Phys. Proc. Suppl. 140, 323 (2005).

[17] S. Aoki and O. Bar, Phys. Rev. D 70, 116011 (2004).

[18] S. R. Sharpe and J. M. S. Wu, hep-lat/0411021

[19] A. M. Abdel-Rehim, R. Lewis and R. M. Woloshyn, hep-lat/0503007

[20] S. R. Beane and M. J. Savage, Phys. Rev. D 68, 114502 (2003)

[21] B. C. Tiburzi, hep-lat/0501020

[22] R. Frezzotti and G. C. Rossi, Nucl. Phys. Proc. Suppl. 128, 193 (2004).

[23] K. Symanzik, Nucl. Phys. B 226, 187 (1983a); B 227, 205 (1983b).

[24] E. Jenkins and A. V. Manohar, UCSD-PTH-91-30, talk presented at the Workshop on Effective Field Theories of the Standard Model, Dobogoko, Hungary, Aug 1991.

[25] E. Jenkins and A. V. Manohar, Phys. Lett. B 255, 558 (1991).

[26] E. Jenkins, Nucl. Phys. B 368, 190 (1992).

[27] J. N. Labrenz and S. R. Sharpe, Phys. Rev. D 54, 4595 (1996).

[28] T. R. Hemmert, B. R. Holstein and J. Kambor, J. Phys. G 24, 1831 (1998).

[29] B. C. Tiburzi and A. Walker-Loud, hep-lat/0501018

[30] A. Manohar and H. Georgi, Nucl. Phys. B 234, 189 (1984). 
[31] V. Bernard, N. Kaiser, and U. G. Meissner, Z. Phys. C 60, 111 (1993).

[32] R. F. Lebed, Nucl. Phys. B 430, 295 (1994).

[33] R. F. Lebed, Phys. Lett. B 329, 479 (1994).

[34] M. K. Banerjee and J. Milana, Phys. Rev. D 52, 6451 (1995).

[35] B. Borasoy and U. G. Meissner, Annals Phys. 254, 192 (1997).

[36] T. Becher and H. Leutwyler, Eur. Phys. J. C 9, 643 (1999).

[37] M. Frink and U. G. Meissner, JHEP 0407, 028 (2004).

[38] A. Walker-Loud, Nucl. Phys. A 747, 476 (2005).

[39] B. C. Tiburzi and A. Walker-Loud, Nucl. Phys. A 748, 513 (2005).

[40] B. C. Tiburzi, Phys. Rev. D 71, 034501 (2005).

[41] B. C. Lehnhart, J. Gegelia, and S. Scherer, hep-ph/0412092

[42] S. R. Beane, Nucl. Phys. B 695, 192 (2004).

[43] G. Munster, C. Schmidt and E. E. Scholz, Europhys. Lett. 86, 639 (2004) arXiv:hep-lat/0402003. 